\title{
SPITZER SAGE SURVEY OF THE LARGE MAGELLANIC CLOUD. III. STAR FORMATION AND 1000 NEW CANDIDATE YOUNG STELLAR OBJECTS
}

B. A. Whitney ${ }^{1}$, M. Sewilo ${ }^{2}$, R. Indebetouw ${ }^{3}$, T. P. Robitaille ${ }^{4}$, M. MeiXner $^{2}$, K. Gordon ${ }^{5}$, M. R. Meade ${ }^{6}$, B. L. Babler $^{6}$, J. Harris ${ }^{5}$, J. L. Hora ${ }^{7}$, S. Bracker ${ }^{6}$, M. S. Povich ${ }^{6}$, E. B. Churchwell ${ }^{6}$, C. W. Engelbracht ${ }^{5}$, B-Q For ${ }^{5,8}$, M. Block $^{5}$, K. Misselt ${ }^{5}$, U. Vijh ${ }^{2}$, C. Leitherer ${ }^{2}$, A. Kawamura ${ }^{9}$, R. D. Blum ${ }^{10}$, M. Cohen ${ }^{11}$, Y. Fukui ${ }^{9}$, A. Mizuno ${ }^{9}$, N. Mizuno $^{9}$, S. Srinivasan ${ }^{12}$, A. G. G. M. Tielens ${ }^{13}$, K. VolK $^{14}$, J-P. Bernard ${ }^{15}$, F. Boulanger ${ }^{16}$, J. A. Frogel ${ }^{17}$, J. Gallagher ${ }^{6}$, V. Gorjian ${ }^{18}$, D. Kelly ${ }^{5}$, W. B. LAtTer ${ }^{19}$, S. Madden ${ }^{20}$, F. Kemper ${ }^{21}$, J. R. Mould ${ }^{10}$, A. Nota ${ }^{2}$, M. S. Oey ${ }^{22}$, K. A. OlSEN ${ }^{23}$, T. Onishi ${ }^{9}$, R. Paladini ${ }^{24}$, N. Panagia ${ }^{2}$, P. Perez-Gonzalez ${ }^{5}$, W. Reach ${ }^{24}$, H. Shibai ${ }^{9}$, S. Sato ${ }^{9}$, L. J. Smith ${ }^{2,25}$,

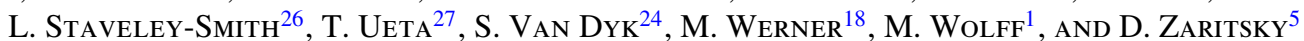

${ }^{1}$ Space Science Institute, 4750 Walnut St. Suite 205, Boulder, CO 80301, USA; bwhitney@ spacescience.org, wolff@ spacescience.org

${ }^{2}$ Space Telescope Science Institute, 3700 San Martin Way, Baltimore, MD 21218, USA; sewilo@stsci.edu, meixner@stsci.edu, vijh@stsci.edu, leitherer@stsci.edu, nota@stsci.edu, panagia@stsci.edu, and 1smith@stsci.edu

${ }^{3}$ Department of Astronomy, University of Virginia, P.O. Box 3818, Charlottesville, VA 22903, USA; remy@ virginia.edu ${ }^{4}$ School of Physics and Astronomy, University of St Andrews, North Haugh, KY16 9SS, St Andrews, UK; tr9@st-andrews.ac.uk ${ }^{5}$ Steward Observatory, University of Arizona, 933 North Cherry Ave., Tucson, AZ 85719, USA; kgordon@as.arizona.edu, cengelbracht@as.arizona.edu, biqing@email.arizona.edu, kmisselt@as.arizona.edu,pgperez@as.arizona.edu,jharris@as.arizona.edu, dkelly@as.arizona.edu, and dennis@fishingholes.as.arizona.edu

${ }^{6}$ Department of Astronomy, 475 North Charter St., University of Wisconsin, Madison, WI 53706, USA; meade@ sal.wisc.edu, brian@sal.wisc.edu, ebc@astro.wisc.edu,jsg@astro.wisc.edu, povich@astro.wisc.edu, and s_bracker@hotmail.com

${ }^{7}$ Center for Astrophysics, 60 Garden St., MS 67, Harvard University, Cambridge, MA 02138, USA; jhora@ cfa.harvard.edu

8 Department of Astronomy, University of Texas at Austin, 1 University Station, C1400, Austin, TX 78712, USA; biqing@astro.as.utexas.edu ${ }_{9}^{9}$ Department of Astrophysics, Nagoya University, Chikusa-ku, Nagoya 464-8602, Japan; fukui@a.phys.nagoya-u.ac.jp, kawamura@a.phys.nagoya-u.ac.jp, mizuno@a.phys.nagoya-u.ac.jp, norikazu@a.phys.nagoya-u.ac.jp, ohnishi@a.phys.nagoya-u.ac.jp, shibai@nagoya-u.jp, and ssato@z.phys.nagoya-u.ac.jp ${ }^{10}$ NOAO, P.O. Box 26732, Tucson AZ 85726-6732, USA; jmould@ noao.edu, rblum@ noao.edu

${ }^{11}$ Radio Astronomy Laboratory, 601 Campbell Hall, University of California at Berkeley, Berkeley, CA 94720, USA; mcohen@ astro.berkeley.edu

12 Department of Physics and Astronomy, Johns Hopkins University, Homewood Campus, Baltimore, MD 21218, USA; sundar@pha.jhu.edu ${ }^{13}$ NASA Ames Research Center, SOFIA Office, MS 211-3, Moffet Field, CA 94035, USA; atielens@arc.nasa.gov ${ }^{14}$ Gemini Observatory, 670 North A'ohuku Place, Hilo, HI 96720, USA; kvolk@ gemini.edu

15 Centre d' Étude Spatiale des Rayonnements, CNRS, 9 av. du Colonel Roche, BP 4346, 31028 Toulouse, France; Jean-Philippe.Bernard@cesr.fr

16 Astrophysique de Pari, Institute (IAP), CNRS UPR 341, 98bis, Boulevard Arago, Paris, F-75014, France; Francois.Boulanger@ias.u-psud.f ${ }^{17}$ AURA, Inc., 1200 New York Ave. NW, Suite 350, Washington D.C. 20005, USA; jfrogel@aura-astronomy.org

18 Jet Propulsion Lab, 4800 Oak Grove Dr., MS 264-767, Pasadena, CA 91109, USA; varoujan.gorjian@jpl.nasa.gov, mwerner@ sirtfweb.jpl.nasa.gov

${ }^{19}$ Caltech, NASA Herschel Science Center, MS 100-22, Pasadena, CA 91125, USA; latter@ipac.caltech.edu ${ }^{20}$ Service dAstrophysique CEA, Saclay, 91191 Gif Sur Yvette Cedex, France; smadden@cea.fr

21 Jodrell Bank Centre for Astrophysics, University of Manchester, M13 9PL, Manchester, UK; f.kemper@manchester.ac.uk

22 Department of Astronomy, University of Michigan, 830 Dennison Bldg., Ann Arbor, MI 48109, USA; msoey@umich.edu ${ }^{23}$ Cerro Tololo Interamerican Observatory, Casilla 603, La Serena, Chile; kolsen@ ctio.noao.edu

${ }^{24}$ Spitzer Science Center, California Institute of Technology, 220-6, Pasadena, CA, 91125, USA; reach@ipac.caltech.edu, vandyk@ipac.caltech.edu ${ }^{25}$ Department of Physics and Astronomy, University College London, Gower Street, London WC1E 6BT, UK

${ }^{26}$ Australia Telescope National Facility, CSIRO, P.O. Box 76, Epping NSW 1710, Australia; Lister.Staveley-Smith@csiro.au

27 Department of Physics and Astronomy, University of Denver, Denver, CO 80208, USA; tueta@ du.edu Received 2007 June 20; accepted 2008 April 1; published 2008 May 27

\section{ABSTRACT}

We present $\sim 1000$ new candidate Young Stellar Objects (YSOs) in the Large Magellanic Cloud selected from Spitzer Space Telescope data, as part of the Surveying the Agents of a Galaxy's Evolution (SAGE) Legacy program. The YSOs, detected by their excess infrared (IR) emission, represent early stages of evolution, still surrounded by disks and/or infalling envelopes. Previously, fewer than 20 such YSOs were known. The candidate YSOs were selected from the SAGE Point Source Catalog from regions of color-magnitude space least confused with other IR-bright populations. The YSOs are biased toward intermediate- to high-mass and young evolutionary stages, because these overlap less with galaxies and evolved stars in color-magnitude space. The YSOs are highly correlated spatially with atomic and molecular gas, and are preferentially located in the shells and bubbles created by massive stars inside. They are more clustered than generic point sources, as expected if star formation occurs in filamentary clouds or shells. We applied a more stringent color-magnitude selection to produce a subset of "high-probability" YSO candidates. We fitted the spectral-energy distributions (SEDs) of this subset and derived physical properties for those that were well fitted. The total mass of these well-fitted YSOs is $\sim 2900 M_{\odot}$ and the total luminosity is $\sim 2.1 \times 10^{6} L_{\odot}$. By extrapolating the mass function with a standard initial mass function and integrating, we calculate a current star-formation rate of $\sim 0.06 M_{\odot} \mathrm{yr}^{-1}$, which is at the low end of estimates based on total ultraviolet and IR flux from the galaxy $\left(\sim 0.05-0.25 M_{\odot} \mathrm{yr}^{-1}\right)$, consistent with the expectation that our current YSO list is incomplete. Follow-up spectroscopy and further data mining will better separate the different IR-bright populations and likely increase the estimated number of YSOs. The full YSO list is available as electronic tables, and the SEDs are available as an electronic figure for further use by the scientific community.

Key words: circumstellar matter - galaxies: dwarf - infrared: stars - Magellanic Clouds - stars: formation - stars: pre-main sequence

Online-only material: extended figure set, machine-readable and VO tables 


\section{INTRODUCTION}

The Large Magellanic Cloud (LMC) holds an advantageous position for studying extragalactic star formation. Its proximity, $50 \mathrm{kpc}$ (e.g., Panagia 2005), permits individual Young Stellar Objects (YSOs) to be identified with the $<2^{\prime \prime}(<0.5 \mathrm{pc})$ resolution available in the optical and near-infrared (IR) using ground-based telescopes, and now in the mid-IR with the Spitzer Space Telescope (Werner et al. 2004). The favorable viewing angle of this flattened galaxy $\left(35^{\circ}\right.$, van der Marel \& Cioni 2001) limits line-of-sight confusion and allows correlation of the YSOs with the interstellar medium (ISM) clouds. The LMC may provide insight into the star-formation processes during the epoch of peak star formation in the universe (redshift of $\sim 1.5$, Madau et al. 1996) because the LMC's metallicity $\left(Z \sim 0.3-0.5 Z_{\odot}\right.$; Westerlund 1997$)$ is similar to the mean metallicity of the universe at redshift of $\sim 1.5$ (e.g., Pei et al. 1999). In addition to its value in studying extragalactic star formation, star formation in the LMC is in some ways easier to study than in our own Galaxy since the distances to the YSOs are known. In the following, we highlight some of the previous findings on star formation in the LMC and how the Spitzer Surveying the Agents of a Galaxy's Evolution (SAGE) IR survey (Meixner et al. 2006) can contribute new information:

1. The star-formation history of the LMC can be determined by modeling optical color-magnitude diagrams (CMDs) obtained from telescopes (e.g., the Hubble Space Telescope $(H S T)$ ) that can resolve individual stars (e.g., Gallagher et al. 1996; Olsen 1999; Smecker-Hane et al. 2002). From such studies, we have learned that the disk star-formation rate (SFR) has been relatively constant over the last $\sim 15$ Gyr, while the bar star formation has been much more episodic. Studies of clusters have shown that the LMC appears to be in an active epoch of cluster formation, which began about 3-4 Gyr ago (Da Costa 1991; Hodge 1988). During this time, the SFR has increased by a factor of about 3 (Geha et al. 1998; Holtzman et al. 1997). These studies nicely determine histories and relative rates of star formation. High-resolution IR studies can complement this work by determining the current SFR.

2. The correlation of giant molecular clouds (Fukui et al. 1999, from the NANTEN CO survey) to optical clusters (e.g., Bica et al. 1996) suggests that the clouds are short lived. This is based on the fact that about half of the CO clouds are associated with the youngest stellar activity $(<10 \mathrm{Myr}$ clusters and $\mathrm{H}$ II regions), implying that stellar clusters are actively formed over about $50 \%$ of the cloud lifetime and that the clouds are dissipated on timescales of $\sim 6 \mathrm{Myr}$. Additionally, the $\mathrm{CO}$ and ultraviolet emission are spatially anti-correlated, suggesting that stellar photons disrupt the clouds. A complete census of YSOs can shed further light on these issues by the degree to which YSOs are spatially correlated with ultraviolet and $\mathrm{CO}$ emission. If molecular cloud lifetimes are short, the efficiency of star formation over the short lifetime must be high to obtain a time- and spatially-averaged efficiency of $\sim 1 \%$. Thus, we should expect to see star formation occurring in most of the molecular clouds, as is the case in the solar neighborhood (Hartmann et al. 2001). Twelve of the 55 well-studied molecular clouds show no star-formation activity, based on optical and nearIR observations (Fukui et al. 1999). A mid-IR survey can reveal more deeply embedded star formation not seen in the near-IR, through both increased emission and decreased extinction, and can more accurately determine the percentages of molecular clouds that are forming stars.

3. Targeted high-spatial-resolution optical and near-IR observations with $H S T$ have identified populations of likely pre-main-sequence (MS) stars based on large reddening or near-IR excesses (Walborn et al. 1999; Panagia et al. 2000; Romaniello et al. 2006; Gouliermis et al. 2006). Two of the most well-studied regions in the LMC are 30 Doradus (N157), and the N159/N160 region just south of it. Brandner et al. (2001) present NICMOS near-IR imaging in the filaments near R136 in the 30 Doradus region, mostly pointed at previously identified candidate protostellar objects and knots of star formation (Hyland et al. 1992; Rubio et al. 1992, among others). They identify 24 candidate protostars, including new, faint sources whose luminosities and colors are consistent with $\mathrm{T}$ Tauri stars. The distribution of young sources in the compressed molecular ridge north of R136 supports a model of triggered star formation in this region. LMC star formation may in general be self-propagating through the energetic feedback of stellar winds and supernovae (e.g., Oey \& Massey 1995; Efremov \& Elmegreen 1998a) but this stellar feedback also acts to eventually squelch star formation by dissipating the local ISM (Yamaguchi et al. 2001; Israel et al. 2003). The SAGE IR survey provides a global picture of star formation in the LMC, and can determine if star formation occurs in clusters and supershells, and the importance of local triggering and self-propagation.

4. Star formation in the LMC is likely influenced by its interactions with its neighbors. Recent $H S T$ observations of proper motions in the LMC (Kallivayalil et al. 2006b) and Small Magellanic Cloud (SMC) (Kallivayalil et al. 2006a) suggest that the $3 \mathrm{D}$ velocities of the Clouds are much higher than previously thought, and the Clouds are on their first passage through the Milky Way (Besla et al. 2007). Besla et al. (2007) suggest that the rise in the SFR in the past $3 \mathrm{Gyr}$ (Zaritsky \& Harris 2004) is due to tidal forces as the Clouds approached the Galactic center. It has been proposed that the southeastern arc in the LMC is star formation triggered by the bow shock as the LMC travels through the Milky Way halo (e.g., de Boer et al. 1998). An IR census can address whether the current global rate of star formation is consistent with an enhancement caused by the current periGalactic passage.

Current star formation is most directly studied with IR instrumentation, since stars form from collapsing dusty envelopes which reradiate the absorbed short-wavelength emission at IR wavelengths. Previous large-scale mid-IR surveys have been limited by resolution and sensitivity. The IRAS survey can be used to identify regions of massive star formation but the lowspatial-resolution makes it difficult to characterize the regions and to tell protostars from the associated diffuse ionized gas, necessitating follow-up at 1 arcsec or better resolution. For example, Indebetouw et al. (2004) mapped the compact H II regions using centimeter-wave interferometry, separating them from embedded YSOs. While Midcourse Space Experiment (MSX) $8 \mu \mathrm{m}$ maps of the LMC provided a wealth of new information on the IR population of the LMC (Egan et al. 2001), MSX E band $(21 \mu \mathrm{m})$ sensitivity was insufficient to image any but the brightest regions in the Magellanic Clouds.

The Spitzer SAGE project (Meixner et al. 2006) allows for the first time a global study of star formation in the LMC at high enough resolution to resolve individual cores and protostars at a 
range of mid-IR wavelengths. SAGE is a Legacy project on the Spitzer Space Telescope (Werner et al. 2004), which mapped a $7^{\circ} \times 7^{\circ}$ region of the LMC using the IRAC camera in the 3.6, 4.5, 5.8, and $8.0 \mu \mathrm{m}$ filters (Fazio et al. 2004) and the MIPS camera in the 24, 70, and $160 \mu \mathrm{m}$ filters (Rieke et al. 2004). The survey was done over two epochs with a total observing time of $291 \mathrm{hr}$ with IRAC and $217 \mathrm{hrs}$ with MIPS. The details of the survey are described in Meixner et al. (2006). ${ }^{28}$ In this paper, we use the SAGE first epoch Point Source Catalogs of IRAC and MIPS $24 \mu \mathrm{m}$ that have been merged together with Two Micron All Sky Survey (2MASS) $J H K_{s}$ (1.2, 1.6, and $2.2 \mu \mathrm{m})$ (Skrutskie et al. 2006).

This paper presents a selection of $\sim 1000$ YSO candidates chosen from the SAGE Point Source Catalog based on their IR colors and magnitudes. The current list is incomplete on both the low- and high-mass ends, but should provide a rich dataset for follow-up observations. The candidate YSO list is available as an online table, as is a table of estimated physical properties of selected YSOs, and an electronic figure of all of the spectral-energy distributions (SEDs). In Section 2 we describe our selection method for the YSOs. Section 3 discusses their properties and comparisons to previously known YSOs, and Section 4 summarizes the results.

\section{COLOR-MAGNITUDE SELECTION OF YOUNG STELLAR OBJECTS}

One advantage of studying star formation in the LMC compared to large regions of the Galaxy is that the distance is known and we can use the magnitude in addition to colors to separate YSOs from other populations. In this section we describe our method for selecting the YSOs. We first describe where we expect YSOs to lie in color-magnitude space based on radiation transfer models (Section 2.1). Next we describe the SAGE Catalog from which the YSOs will be selected, and identify known populations such as galaxies and evolved stars in the CMDs (Section 2.2). Finally, in Section 2.3 we describe the color-magnitude selection of the YSOs, and further culling and quality checks.

\subsection{The Expected Colors and Magnitudes of YSOs}

YSOs are typically surrounded by dusty envelopes and disks. In their early stages of evolution, the envelopes are opaque and relatively cool, and absorb most of the central stellar radiation, re-emitting in the IR at the temperature of the dust. As these sources evolve, the envelopes and disks disperse and emit less IR radiation. An ensemble of YSOs of different evolutionary stages and masses will span a large range in IR colors. Robitaille et al. (2006) computed a grid of 20,000 2D radiation transfer models intended to cover the full range of stellar masses and evolutionary stages of YSOs. Each model produces SEDs at ten inclinations and 50 apertures. The models accurately compute scattered and thermal emission from dust in a physically plausible geometry, which consists of a stellar source that illuminates a dusty disk, envelope, and bipolar cavity (Whitney et al. 2003a, 2003b). The models are based on observations and theory of known Galactic star-forming regions, and have been used to fit SEDs of YSOs in the Taurus molecular cloud (Robitaille et al. 2007), the G34.4+0.2 massive star-forming

28 Catalogs that combine 2MASS $J H K_{s}$, IRAC and MIPS 24 are available at the Spitzer Science Center Website

http://ssc.spitzer.caltech.edu/legacy/all.html. region (Shepherd et al. 2007), the M16 star-forming region (Indebetouw et al. 2007), several newly discovered massive YSOs formed in bubbles (Watson et al. 2008), as well as YSOs in the SMC (Simon et al. 2007).

We can use the grid of models to estimate where the LMC YSOs might lie in color-magnitude space. As described in Robitaille et al. (2006), we can create a model CMD of the LMC by weighting models of the grid to produce a standard Initial Mass Function (IMF) for the stellar masses (Kroupa 2001), and a constant SFR beginning two million years ago. Most of the YSOs we are sensitive to will be much younger than this and thus there is no need to go further back in time. We define the age of a source as the time since the pre-MS contraction began. For the IMF, we use average slopes of -1.3 for stellar mass greater than 0.5 , and -0.3 for mass less than 0.5 and a mass range of $0.08-50 M_{\odot}$ (Kroupa 2001). We then scaled the resulting model fluxes to the distance of the LMC and applied the same sensitivity limits as the SAGE data. A similar comparison of our model CMDs and CCDs in the Perseus starformation region found good agreement between the models and data (Harvey et al. 2007). The resulting distribution of model YSOs is shown in Figure 1. The CMDs are displayed as Hess diagrams (2D histograms where the number density is represented by the brightness of each pixel), but in three colors. The models were divided into three ranges of evolutionary stage (Figure 1(a)) and mass bins (Figure 1(b)). In Figure 1(a), the red image in each panel corresponds to the youngest YSOs (Stage I: embedded in an infalling envelope); the green image to stars surrounded by opaque disks (Stage II); and blue to stars surrounded by optically thin disks (Stage III). The stages were defined in Robitaille et al. (2006) based on comparison to the observationally-derived class scheme as follows: Stage I objects have an envelope infall rate $\dot{M}_{\text {env }} / M_{\star}>10^{-6} \mathrm{yr}^{-1}$, Stage II objects have $\dot{M}_{\text {env }} / M_{\star}<10^{-6} \mathrm{yr}^{-1}$ and $M_{\text {disk }} / M_{\star}>$ $10^{-6}$, and Stage III objects have $\dot{M}_{\text {env }} / M_{\star}<10^{-6} \mathrm{yr}^{-1}$ and $M_{\text {disk }} / M_{\star}<10^{-6}$. For each evolutionary stage, the brightness of a pixel increases with the density of the models. The three evolutionary stages are combined into a three-color image. Pixels with overlapping stages have blended colors. Shades of gray (white to black) indicate similar numbers of sources at all evolutionary stages. The bottom-left panel of Figure 1(a) shows that the evolutionary stages overlap at [3.6]- [8.0] but are better separated at [8.0]-[24]. Figure 1(b) shows how stellar masses segregate with magnitude. Here the red, green, and blue images correspond to three mass ranges: $M_{\star}<5 M_{\odot}$ (red); $5 M_{\odot}<M_{\star}<10 M_{\odot}$ (green); and $M_{\star}>10 M_{\odot}$ (blue). This shows that our current catalog is not very sensitive to low-mass $\left(<5 M_{\odot}\right)$ sources, though future catalogs based on our twoepoch mosaic photometry will go deeper. A few features of the CMDs are worth explaining briefly. The lack of models at upper left (luminous and less red) are due to our inclusion of an ambient density outside the disk and a rotationally-flattened envelope (if present) that has a large column density in the high-mass models (we neglected the effect of stellar winds and photo-evaporation on the ambient medium). The curvature on the right side of the plots, most noticeable in Figure 1(b) is due to the fact that as a source of a given mass evolves, it becomes brighter and less red at near-IR and IRAC wavelengths because of the lower-envelope extinction and emission.

These models were developed based on our knowledge of nearby low-mass star-formation regions, and were tested by comparing known physical properties of several Taurus sources to those derived from SED fitting (Robitaille et al. 2007). 

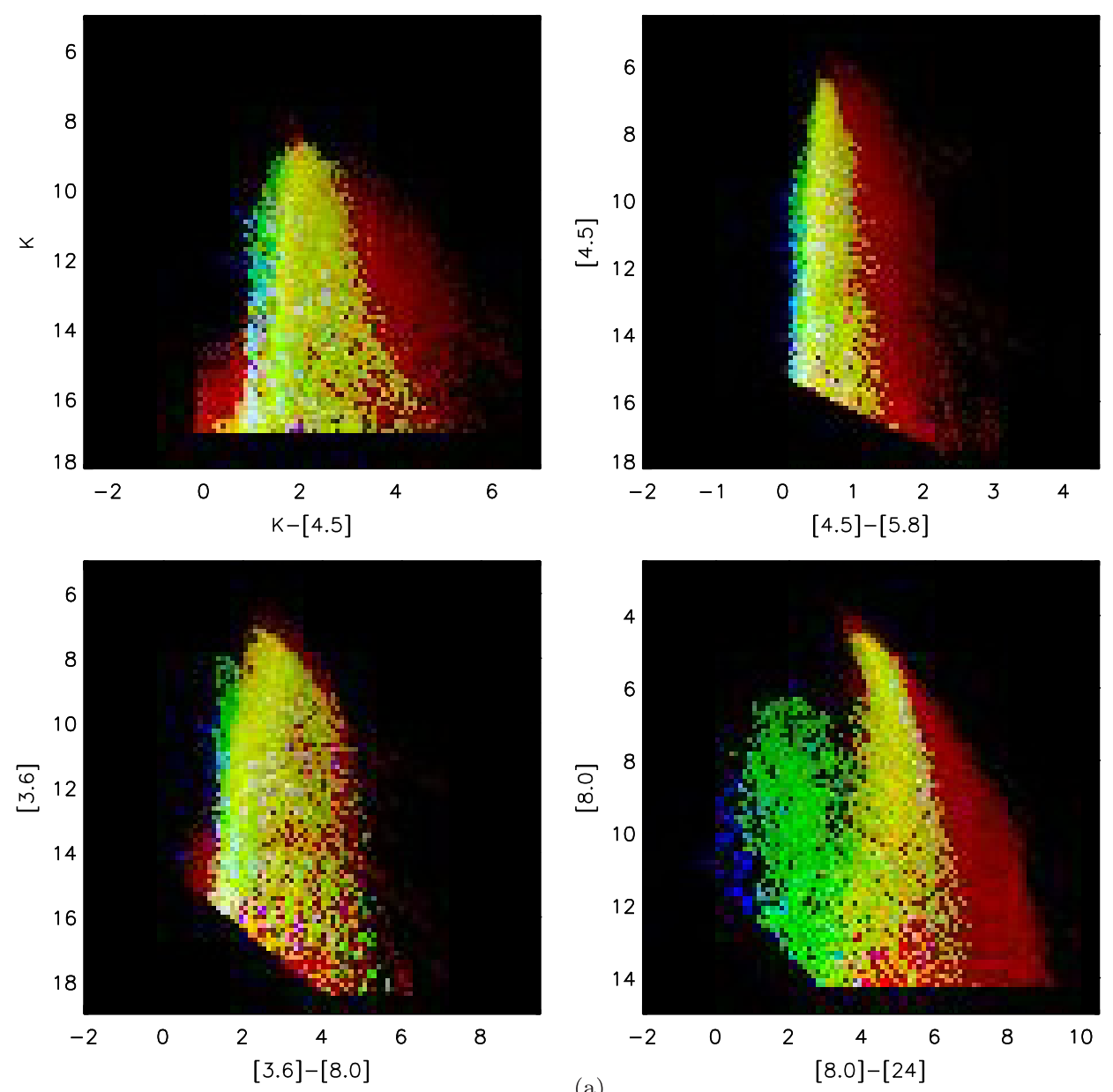

Figure 1. CMDs from a grid of YSO models scaled to a standard IMF and SAGE sensitivity limits. (a) Each panel is a three-color combination of a red, green, and blue image representing three stages of evolution: the red image corresponds to the youngest embedded YSOs; the green image to YSOs surrounded by opaque disks; and the blue to stars surrounded by optically thin disks. The colors blend in regions where the evolutionary stages overlap. (b) The combined red, green, and blue images correspond to three mass ranges: $M_{\star}<5 M_{\odot}\left(\right.$ red) $; 5 M_{\odot}<M_{\star}<10 M_{\odot}$ (green); and $M_{\star}>10 M_{\odot}$ (blue).

For the high-mass models, we used parameters consistent with disk/envelope masses and radii derived from observations (see references in Robitaille et al. 2007). Some potential inadequacies in the models applied to the LMC are the following. (1) The models assume a single central source illuminates the circumstellar geometry. At the distance to the LMC, the $2^{\prime \prime}$ beam of IRAC subtends $\sim 0.5 \mathrm{pc}$, and the larger beam at $24 \mu \mathrm{m}$ subtends $\sim 1.5 \mathrm{pc}$. Thus, our "point sources" may contain more than one object. As discussed in Section 3.4, we will likely underestimate the total mass of the "source" if it is a cluster instead of a single YSO. (2) The models do not include polycyclic aromatic hydrocarbon (PAH) emission which can be important in sources with hot central stars, though the contribution is lower and often non-existent in more embedded sources (Gibb et al. 2000; Keane et al. 2001), which we are more sensitive due to our color-magnitude selections (Section 2.3.1). (3) The dust opacity and scattering properties are based on models and data of Galactic extinction (Weingartner \& Draine 2001; Indebetouw et al. 2005). Although there are known differences between Galactic and LMC dust properties (e.g., Weingartner $\&$ Draine 2001), these differences are less pronounced in the IR (e.g., Draine 2003). We rescale the YSO model infall rates and disk masses to account for the lower dust-to-gas ratio in the LMC (0.005, compared to 0.01 in the Galaxy), based on Gordon et al. (2005)'s LMC average sample. (4) The high-mass models may have contributions to the SED from gas in the inner disk, which will be hotter than the dust. In addition, photo-evaporation from the disks around high-mass stars likely increases the scale height of the disk, contributing more IR emission (Robberto et al. 2002; Hollenbach et al. 1994). As in point (2) our final YSO list contains more embedded sources in which the envelope dominates the IR flux and therefore this inadequacy is less important. However, we are currently improving our models to include both PAH emission and more accurate disk models for high-mass sources.

Simon et al. (2007) used SED fitting to select YSOs in the N66 region of the SMC and compared these to color-magnitude selections. SED fitting finds more sources than the usual twocolor or single color-magnitude selections because it uses more information (the full SED). This works well in star-formation regions where more of the IR-excess sources are likely to be YSOs and where individual sources can be examined carefully. For a galaxy-wide search of YSOs, SED-fitting has not yet been proven to uniquely select YSOs from other IR-excess populations (dusty evolved stars and galaxies). Therefore in this paper, we will use an empirical method to select the YSO candidates, looking for regions of color-magnitude space less occupied by other populations (e.g., galaxies and evolved stars). 

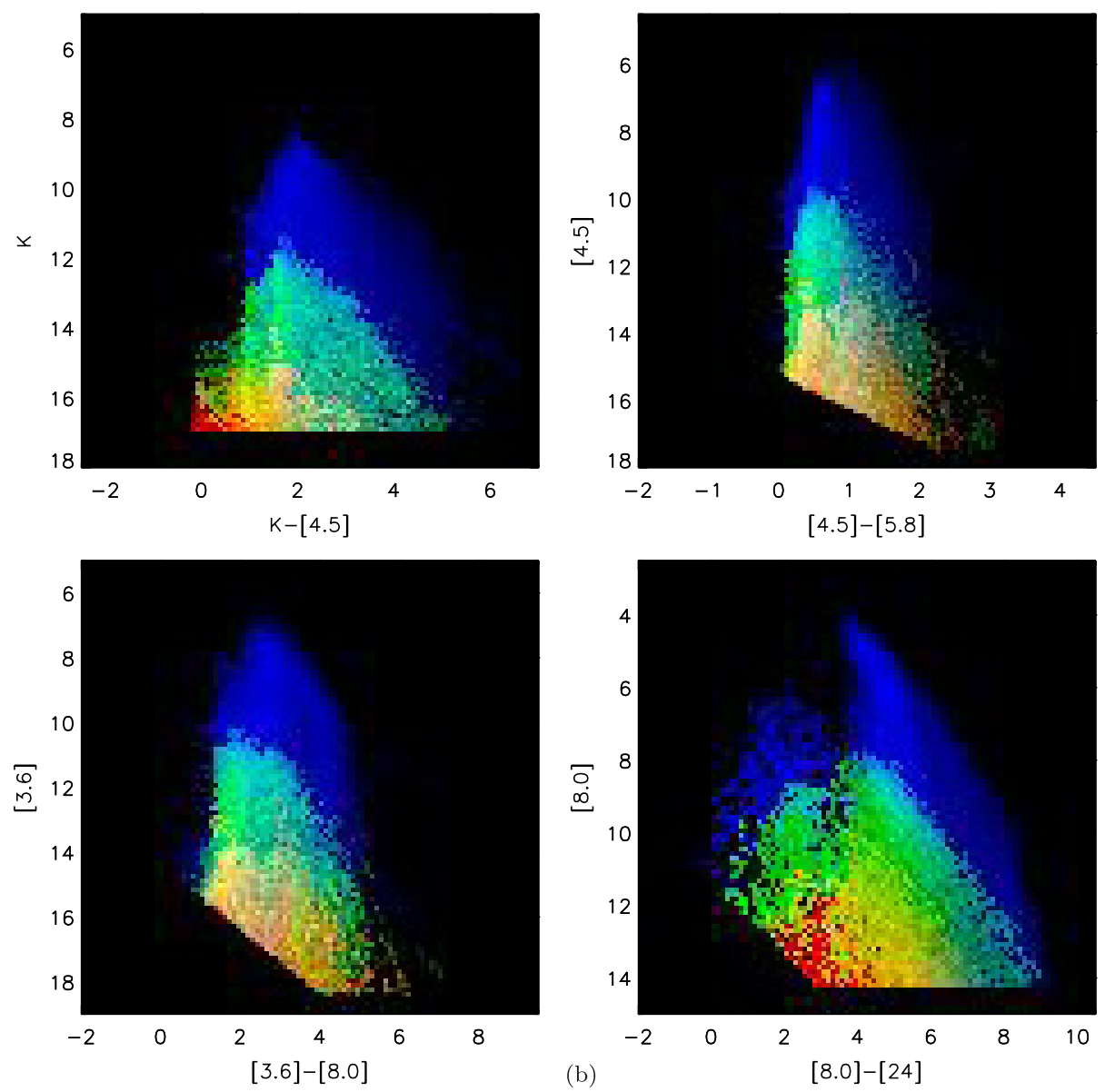

Figure 1. (Continued)

Once the YSO candidates have been identified, we will use the YSO models to help interpret their physical properties.

\subsection{The SAGE Catalog, and Infrared Stellar Populations}

The SAGE Point Source Catalog used in this paper was generated from data taken during our first epoch of observing. The IRAC data were taken on 2005 July $15-26$ and the MIPS data on 2005 July $27-$ August 3 . The IRAC data from the first epoch consisted of two visits on the sky each at 0.4 and $10.4 \mathrm{~s}$ exposure times in HDR mode. A total of about 37,500 frames per band cover the $7^{\circ} \times 7^{\circ}$ region of the LMC. The MIPS $24 \mu \mathrm{m}$ first epoch observations had ten visits on the sky, with a total exposure time of $30 \mathrm{~s}$. MIPS 70 and $160 \mu \mathrm{m}$ data were also taken but we present only the $24 \mu \mathrm{m}$ data in this paper, as its higher resolution is more readily matched with the 2MASS and IRAC Point Source Catalogs.

The data processing is described in detail in Meixner et al. (2006). To briefly summarize, the IRAC data were processed using the University of Wisconsin's pipeline. ${ }^{29}$ After artifacts from the images were removed, photometry was performed on each IRAC frame using a modified version of DAOPHOT (Stetson 1987). A second pass of photometry on the residual images improved the fluxes and removed false sources, especially in regions with complicated background emission. The fluxes

29 Documentation is available at

http://www.astro.wisc.edu/glimpse/docs.html. were merged over multiple visits on the sky and across wavelengths, to produce a catalog. At the same time, the lists were merged with the 2MASS ( $J, H$, and $K_{s}$ ) Catalog (Skrutskie et al. 2006). We did not remove cosmic rays from the images prior to photometry, so to ensure reliability, sources were required to be detected multiple times and at multiple wavelengths. To ensure accuracy, fluxes were nulled if the signal-to-noise was $<6$ in the [3.6], [4.5], and [5.8] bands, and $<10$ in the [8.0] band. Future versions of the pipeline will perform photometry on mosaic images with cosmic rays removed and will be more complete at faint magnitudes than the current source list.

The MIPS data were processed using the MIPS Data Analysis Tool version 3.02 (DAT; Gordon et al. 2005). Additional processing steps removed residual instrumental signatures (details in Meixner et al. 2006). Point source photometry was performed on the mosaic images using the point-spread function (PSF)-fitting program StarFinder (Diolaiti et al. 2000). Similar to the IRAC processing, iterations on the residual image were done, in this case to produce a background-subtracted image on which final photometry was done. Three separate MIPS Catalogs were produced, for the 24, 70, and $160 \mu \mathrm{m}$ bands, but were not merged because the angular resolution between them differs substantially.

The resulting IRAC+2MASS and MIPS $24 \mu \mathrm{m}$ Catalogs used in this paper are available at the Spitzer Science Center Website. ${ }^{30}$ The two catalogs were cross matched using a

\footnotetext{
${ }^{30} \mathrm{http}: / /$ ssc.spitzer.caltech.edu/legacy/all.html.
} 

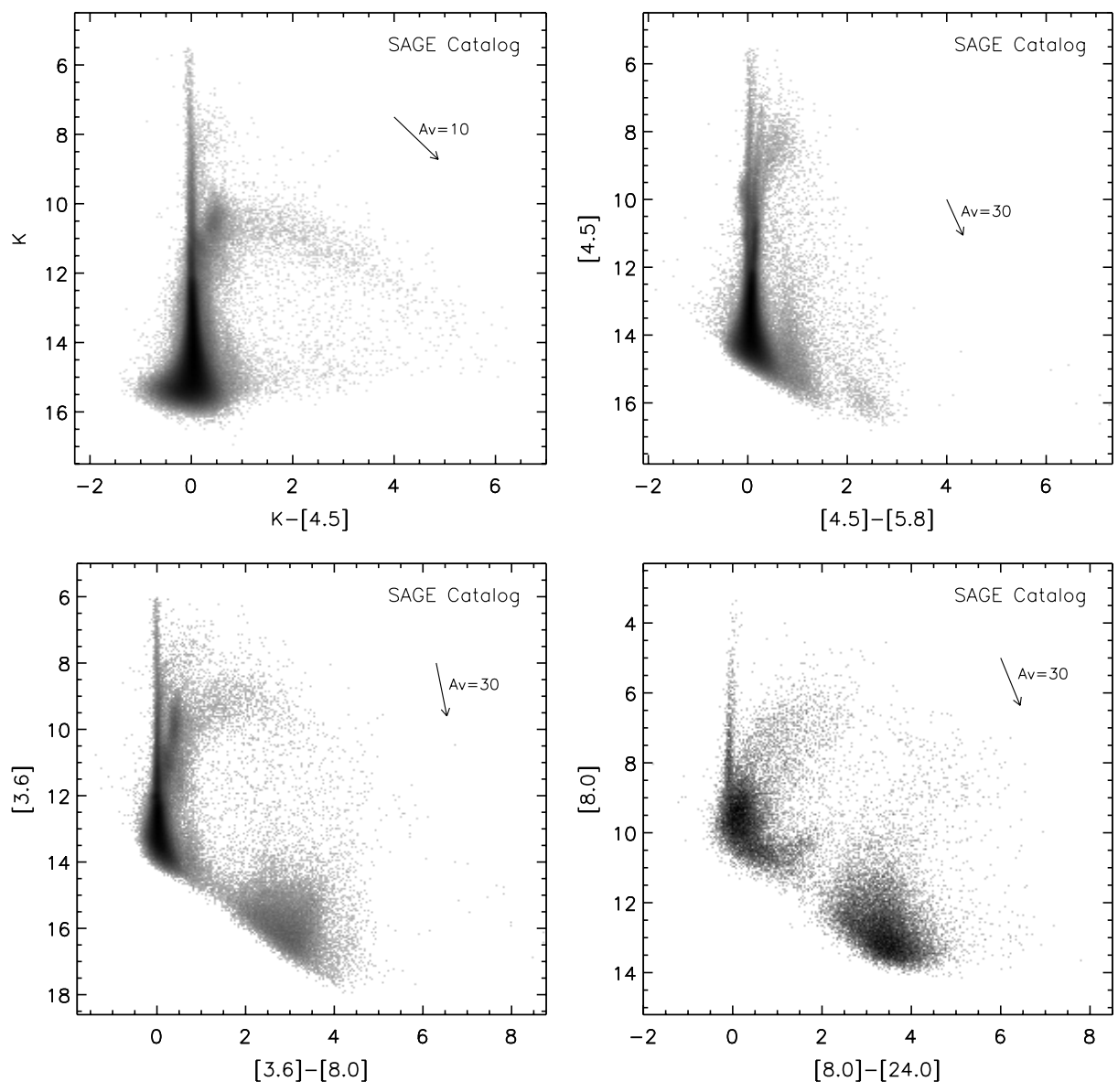

Figure 2. Selected CMDs from the SAGE Point Source Catalog.

database program at STScI (Meixner et al. 2006). We include the cross-matched sources in our list if the distances between the matched sources are $\leqslant 1^{\prime \prime}$.

Figure 2 shows sample CMDs of the Catalog, using the same sequences as Figure 1. As in Figure 1, these are displayed as Hess diagrams, that is, with brightness of each pixel corresponding to the density of sources. The various stellar populations are described in detail in Blum et al. (2006).

Different populations can be identified in the CMDs. These include carbon-rich (C-rich) asymptotic giant branch stars (AGBs), oxygen-rich (O-rich) AGBs, and extreme AGBs (Srinivasan et al. 2008; Blum et al. 2006); planetary nebulae (PNs; Hora et al. 2008), and galaxies. Figure 3 shows these populations overlayed on the Catalog (in gray scale) and the YSO models (in orange-tinted gray scale). The YSO models are displayed transparently so the overlap between the catalog and YSO models is tinted. The known sources are overlayed and block the regions underneath. The $\mathrm{C}$-rich and O-rich AGBs were color-magnitude selected using Equations (5), (6), and (7) from Cioni et al. (2006), assuming a metallicity of $[\mathrm{M} / \mathrm{H}]$ $\sim 0.38$. Extreme AGBs were color-magnitude selected assuming $J-[3.6] \geqslant 3$ in a [3.6] versus [3.6] $-J$ diagram (Blum et al. 2006). The known AGBs in Figure 3 were taken from van Loon et al. (1999). PNs were selected using the Leisy et al. (1997) catalog of PNe in the LMC. Of the 280 sources in the Leisy catalog, 213 had identifications within $1.5^{\prime \prime}$ of the SAGE Point Source Catalog position and these were selected for plotting, using the SAGE Catalog fluxes.
The "empty-field" data shown in Figure 3 were taken in the four corner edges of the SAGE survey region, covering 1.57 square degrees in total. We fitted the SEDs of these outer-region data with stellar atmosphere models from Brott \& Hauschildt (2005) and Kurucz (1993) using a linear regression fitter (Robitaille et al. 2007), and removed well-fitted sources, leaving 979 sources with IR excesses. These remaining sources consist mostly of galaxies, and evolved Milky Way and LMC stars. As a check, we compared with data from the Spitzer SWIRE Legacy survey field centered on the Lockman Hole (Lonsdale et al. 2004). These fall in the same area on the CMD as our empty-field region. While the SWIRE data are much more numerous, they are processed with different techniques, and could lead to slight biases in colors, particularly in the extended sources, so we show the empty-field region in the plots. However, we used the empty field, the SWIRE data, and the density of the catalog sources in determining the boundaries between the YSOs and galaxies, since the SWIRE and SAGE Catalog data are much more numerous.

\subsection{Selection of YSO Candidates}

\subsubsection{Color-Magnitude Selection of YSOs}

Our approach in this paper is to select YSO candidates from regions of color-magnitude space occupied predominantly by YSOs, and less so by other stellar populations. In the process, we have to discard YSOs that overlap with a high density of other stellar populations. Figure 3 shows regions in color-magnitude 
space we have selected, redward of the purple lines. Note that no regions of color-magnitude space are completely devoid of other populations so we expect some contamination in our YSO list, but by number these are expected to be less numerous than YSOs. The lower-left panel of Figure 3(d) can be compared to Bolatto et al. (2007)'s color-magnitude selection of YSOs in the Spitzer $\mathrm{S}^{3} \mathrm{MC}$ survey of the SMC. They chose sources with [5.8]-[8.0] > 1.2 and [5.8] brighter than the galaxydominated region. Our region is similar, though rotated a little. We also include as many color-magnitude selections as possible to increase the number of selected sources. As Figure 3 shows, we select YSOs that are brighter than galaxies, redder than most evolved sources, and in some cases fainter than extreme AGB sources. Figure 1 shows that this selection biases our YSO list toward younger evolutionary stages and intermediate to high mass. While on the subject of biases, we note that the currently released SAGE Catalogs do not include MIPS 70 or $160 \mu \mathrm{m}$ datapoints, so we are not sensitive to the very youngest sources which may be detected only at MIPS wavelengths. In addition, sources extended at IRAC wavelengths are not in the Point Source Catalog. Often these are the most massive sources as they illuminate large volumes. Thus, while we are sensitive to a relatively high mass and young sources, we are likely missing the most massive YSOs.

To select the YSO candidates, we applied the following color-magnitude selection criteria to the "universal table" via a database query:

$\begin{aligned} & {[3.6]<6.76+1.10 \times([3.6]-[24]) } \\ \text { or } & {[4.5]<7.26+1.02 \times([4.5]-[24]) } \\ \text { or } & {[3.6]<10.6+3.50 \times([3.6]-[4.5]) } \\ \text { or } & {[3.6]-[4.5]>1.5 } \\ \text { or } & {[3.6]-[8.0]>3.5 } \\ \text { or } & {[3.6]-[8.0]>4.5 } \\ \text { or } & {[3.6]-[8.0]>1.5 } \\ \text { and } & {[3.6]<13.5 } \\ \text { or } & {[5.8]<7.83+0.89 \times([5.8]-[24]) } \\ \text { or } & {[8.0]<7.59+1.06 \times([8.0]-[24]) } \\ \text { or } & {[24]<3.72+0.95 \times([8.0]-[24]) } \\ \text { or } & {[4.5]>11.91-2.54 \times([4.5]-[5.8]) } \\ \text { or } & {[8.0]<12.52-0.73 \times([4.5]-[8.0]) } \\ \text { and } & {[8.0]>10.58-1.49 \times([4.5]-[8.0]) } \\ \text { or } & {[4.5]-[8.0]>3.7 } \\ \text { or } & {[4.5]-[8.0]>2.7 } \\ \text { or } & {[4.5]<11.12+0.94 \times([4.5]-[8.0]) } \\ \text { and } & {[4.5]>11.13-0.89 \times([4.5]-[8.0]) } \\ \text { or } & {[5.8]<10.92+0.89 \times([5.8]-[8.0]) } \\ \text { or } & K>12.5 \\ \text { and } & K-[4.5]>1.5 \\ \text { or } & K>12.5 \\ \text { and } & K-[3.6]>1.0 \\ & \end{aligned}$

spatially correlated with diffuse emission, especially at $24 \mu \mathrm{m}$ (Calzetti et al. 2005), we required that the $24 \mu \mathrm{m}$ diffuse emission near each source be $>0.08 \mathrm{MJy} \mathrm{sr}^{-1}$ for the source to remain in the list. We did not wish to bias the results too much because we are interested in studying the spatial correlation of YSOs with $24 \mu \mathrm{m}$ emission. Thus, we chose a low threshold $\left(0.08 \mathrm{MJy} \mathrm{sr}^{-1}\right)$ to cull on, which removed sources mostly from the outer four corners of the rectangular survey region.

Next, we increased our IRAC [5.8] and IRAC [8.0] errors by $10 \%$ and $30 \%$ respectively based on an empirical analysis of the root-mean-squared variations of the flux measurements between observations (most sources were observed twice). We required that each source have at least three detections among the five IRAC and MIPS24 bands, each with the modified signal-tonoise (flux/error) $>10$. This reduced the source list to $\sim 1250$ sources.

We fitted stellar atmosphere models to all the sources and examined the fits. In cases where a stellar atmosphere could be fitted if one of the data points were missing (except $24 \mu \mathrm{m}$ ), we removed the source from the list. We also fitted YSO models to the remaining sources (Robitaille et al. 2007), and visually examined the SEDs, model fits, the image at each wavelength, and residual images produced by extracting the catalog flux from the mosaic images. In this way, we found some resolved galaxies, bad point-source extractions, mismatched 2MASS and IRAC sources, and other questionable results. This only removed about 50 sources, leaving 1197 sources. We searched

As mentioned above, this query selects regions of colormagnitude space redward of the purple lines in Figure 3 or inside the box in some panels, and performs a logical "OR" of each panel. This means that if a source falls in the box or redward of the purple line in any of these panels, it cannot be explained as one of the identified known populations, except for some of the evolved stars (e.g., PNs) which overlap with YSOs. The resulting initial list of YSO candidates contained 3773 sources.

\subsubsection{Further Culling and Quality Checks of the YSO List}

To obtain a high-quality and reliable list, we performed several more checks and culls. First, since star formation is

and $\quad[3.6]>13.86-0.91 \times([3.6]-[24])$

and $[4.5]>10.79-0.53 \times([4.5]-[24])$

and $[3.6]>13.35-2.41 \times([3.6]-[4.5])$

$[3.6]<13.5$

$[3.6] \geqslant 13.5$

$[3.6]-[8.0] \leqslant 3.5$

$[3.6]>10.5)$

$[5.8]>10.79-0.81 \times([5.8]-[24])$

$[8.0]>11.0-1.33 \times([8.0]-[24])$

$[24]>9.76-1.79 \times([8.0]-[24])$

$[4.5]<9.44+3.57 \times([4.5]-[5.8])$

$[8.0]>28.30-18.29 \times([4.5]-[8.0])$

$[8.0]<8.0$

$[4.5]>24.0-13.0 \times([4.5]-[8.0])$

$[5.8]>16.66-6.60 \times([5.8]-[8.0])$

$K<14.0$

$K-[4.5]<3.5$

$K<13.5$

$K-[3.6]<2.5$.

for asteroids in the list by comparing to our second epoch data taken approximately three months after the first. All of the YSO candidates appear in both lists at the same locations, so are not asteroids. The list of 1197 YSO candidates is shown in Tables 1 and 2. Note that the source designations in this paper differ from the online catalog in that a space has been removed.

\subsubsection{Cross Correlation with Other Catalogs}

We correlated the YSO candidate list with known stellar populations and found 82 PNs (Leisy et al. 1997; Reid \& Parker 2006), two Wolf-Rayet (WR) stars (Breysacher et al. 1999), two emission line stars (Bohannan \& Epps 1974), and 13 carbon 
Table 1

YSO Candidates: Fluxes

\begin{tabular}{|c|c|c|c|c|c|c|c|c|c|c|c|c|}
\hline \multirow[t]{2}{*}{ No. } & \multirow[t]{2}{*}{ IRAC designation } & \multirow{2}{*}{$\begin{array}{c}\text { R.A. (J2000) } \\
(\mathrm{deg})\end{array}$} & \multirow{2}{*}{$\begin{array}{c}\text { Decl. (J2000) } \\
(\mathrm{deg})\end{array}$} & \multirow{2}{*}{$\begin{array}{c}\text { IracMipsDist } \\
(\operatorname{arcsec})\end{array}$} & \multicolumn{8}{|c|}{ Fluxes in mJy } \\
\hline & & & & & $F_{J}$ & $F_{H}$ & $F_{K}$ & $F_{3.6}$ & $F_{4.5}$ & $F_{5.8}$ & $F_{8.0}$ & $F_{24}$ \\
\hline 1 & SSTISAGE1CJ044033.46-683825.0 & 70.139424 & -68.640296 & 0.002 & $0.63(0.05)$ & $0.44(0.07)$ & $0.76(0.08)$ & $1.85(0.06)$ & $2.75(0.16)$ & $4.00(0.11)$ & $5.15(0.2)$ & $8.97(0.16)$ \\
\hline 2 & SSTISAGE1CJ044037.28-690321.6 & 70.155371 & -69.056018 & 0.005 & (1) & & the & $0.80(0.03)$ & $1.16(0.06)$ & $1.63(0.07)$ & $2.19(0.07)$ & $4.72(0.09)$ \\
\hline 3 & SSTISAGE1CJ044139.53-683247.7 & 70.414729 & -68.546605 & 0.005 & $\ldots$ & .. & 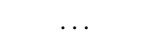 & $0.32(0.01)$ & $0.23(0.02)$ & & $1.98(0.08)$ & $10.00(0.15)$ \\
\hline 4 & SSTISAGE1CJ044254.47-693719.1 & 70.726994 & -69.621985 & 0.009 & $\ldots$ & $\ldots$ & $\ldots$ & $1.29(0.08)$ & $1.71(0.10)$ & $1.91(0.11)$ & $2.27(0.11)$ & $2.34(0.06)$ \\
\hline 5 & SSTISAGE1CJ044304.54-703919.3 & 70.768946 & -70.655388 & 0.007 & $\ldots$ & $\ldots$ & $\ldots$ & $0.28(0.03)$ & 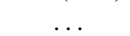 & $1.38(0.14)$ & $3.71(0.44)$ & $7.72(0.19)$ \\
\hline 6 & SSTISAGE1CJ044515.41-690038.9 & 71.314239 & -69.010832 & 0.002 & $\ldots$ & $\ldots$ & $\cdots$ & & $0.72(0.05)$ & $0.79(0.05)$ & $1.78(0.09)$ & $6.08(0.12)$ \\
\hline 7 & SSTISAGE1CJ044629.40-703648.0 & 71.622507 & -70.613360 & 0.001 & $0.47(0.05)$ & $0.78(0.08)$ & $0.82(0.11)$ & $0.71(0.06)$ & $0.73(0.05)$ & $0.93(0.06)$ & $5.01(0.15)$ & $19.81(0.27)$ \\
\hline 8 & SSTISAGE1CJ044659.61-692217.1 & 71.748414 & -69.371421 & 0.006 & $10.02(0.26)$ & $13.49(0.35)$ & $11.39(0.28)$ & $7.18(0.37)$ & $5.42(0.18)$ & $4.43(0.18)$ & $4.82(0.11)$ & $1.76(0.05)$ \\
\hline 9 & SSTISAGE1CJ044716.69-671339.2 & 71.819555 & -67.227560 & 0.004 & & & & $0.41(0.03)$ & $0.52(0.04)$ & $1.16(0.06)$ & $2.89(0.11)$ & $8.86(0.14)$ \\
\hline 10 & SSTISAGE1CJ044717.51-690930.2 & 71.822965 & -69.158402 & 0.004 & $0.59(0.06)$ & $0.70(0.09)$ & $1.12(0.10)$ & $2.99(0.20)$ & $2.93(0.18)$ & $14.84(0.55)$ & $45.78(0.78)$ & $918.6(4.81)$ \\
\hline
\end{tabular}

(This table is available in its entirety in machine-readable and Virtual Observatory (VO) forms in the online journal. A portion is shown here for guidance regarding its form and content.) 
Table 2

YSO Candidates: Magnitudes

\begin{tabular}{|c|c|c|c|c|c|c|c|c|c|c|c|}
\hline \multirow[t]{2}{*}{ No. } & \multirow{2}{*}{$\begin{array}{c}\text { R.A. (J2000) } \\
(\mathrm{deg})\end{array}$} & \multirow{2}{*}{$\begin{array}{c}\text { Decl. (J2000) } \\
\text { (deg) }\end{array}$} & \multicolumn{8}{|c|}{ Magnitudes } & \multirow[t]{2}{*}{ Class. $^{\mathrm{a}}$} \\
\hline & & & $J$ & $H$ & $K_{s}$ & [3.6] & [4.5] & [5.8] & {$[8.0]$} & [24] & \\
\hline 1 & 70.139424 & -68.640296 & $16.01(0.08)$ & $15.91(0.16)$ & $14.85(0.12)$ & $12.94(0.03)$ & $12.04(0.06)$ & $11.16(0.03)$ & $10.22(0.04)$ & $7.26(0.02)$ & YSO \\
\hline 2 & 70.155371 & -69.056018 & $\cdots$ & $\cdots$ & $\cdots$ & $13.85(0.04)$ & $12.97(0.05)$ & $12.14(0.05)$ & $11.15(0.04)$ & $7.95(0.02)$ & YSO_hp \\
\hline 3 & 70.414729 & -68.546605 & $\cdots$ & $\cdots$ & $\cdots$ & $14.83(0.05)$ & $14.74(0.08)$ & $\cdots$ & $11.26(0.04)$ & $7.14(0.02)$ & YSO \\
\hline 4 & 70.726994 & -69.621985 & $\cdots$ & $\cdots$ & $\cdots$ & $13.33(0.07)$ & $12.55(0.07)$ & $11.96(0.06)$ & 11.11(0.05) & $8.72(0.03)$ & YSO_hp \\
\hline 5 & 70.768946 & -70.655388 & $\cdots$ & $\cdots$ & $\cdots$ & $14.99(0.13)$ & $\cdots$ & $12.32(0.11)$ & $10.58(0.13)$ & $7.42(0.03)$ & YSO \\
\hline 6 & 71.314239 & -69.010832 & $\ldots$ & $\cdots$ & $\cdots$ & $\cdots$ & $13.49(0.08)$ & $12.92(0.06)$ & 11.37(0.05) & $7.68(0.02)$ & YSO_hp \\
\hline 7 & 71.622507 & -70.61336 & $16.32(0.13)$ & $15.29(0.12)$ & $14.78(0.14)$ & 13.98(0.09) & $13.48(0.07)$ & $12.75(0.07)$ & $10.25(0.03)$ & $6.40(0.02)$ & YSO \\
\hline 8 & 71.748414 & -69.371421 & $13.00(0.03)$ & $12.20(0.03)$ & $11.92(0.03)$ & 11.47(0.06) & $11.30(0.04)$ & $11.05(0.04)$ & $10.29(0.02)$ & $9.03(0.03)$ & YSO \\
\hline 9 & 71.819555 & -67.22756 & $\cdots$ & $\cdots$ & $\cdots$ & $14.56(0.07)$ & $13.85(0.08)$ & $12.51(0.06)$ & $10.85(0.04)$ & $7.27(0.02)$ & YSO_hp \\
\hline 10 & 71.822965 & -69.158402 & $16.08(0.10)$ & $15.41(0.13)$ & $14.44(0.10)$ & $12.42(0.07)$ & $11.97(0.07)$ & $9.74(0.04)$ & $7.85(0.02)$ & $2.23(0.01)$ & YSO \\
\hline
\end{tabular}

Notes.

a YSO, Young Stellar Object; g, good fit; b, bad fit; PN, Planetary Nebula; Evolved, this category includes Wolf-Rayet, Emission Line, post-AGB, C-rich AGB stars, and cepheids; also labeled are SN87, four probable background galaxies, and two X-ray sources.

(This table is available in its entirety in machine-readable and Virtual Observatory (VO) forms in the online journal. A portion is shown here for guidance regarding its form and content.)

stars (Kontizas et al. 2001) in the list. Interestingly, the WR stars had been highlighted in our visual inspection because they show excess only at $24 \mu \mathrm{m}$, and the $24 \mu \mathrm{m}$ flux is extended. Since WR stars often have large shells, this makes more sense than, e.g., a YSO with a very large disk. Another 70 sources overlap with a list of candidate AGB and post-AGB stars being compiled by A. Ginsburg et al. (2008, in preparation) from the same SAGE dataset based on their SED shapes. These are only candidate-evolved stars, but we conservatively identify them as such in Table 2.

We performed a SIMBAD search on all of the sources and found matches for approximately 80 sources identified as possible evolved stars and galaxies, about 15 of which overlapped with the above lists. Many of these were colormagnitude selected using similar criteria as our YSOs, (e.g., the PN candidates from Egan et al. 2001), so could be YSOs. Only about $25 \%$ of the sources are spectroscopically confirmed, but after examining the images and SEDs, we concluded that many of these have a reasonable likelihood of being evolved stars. This list is a heterogeneous group of possible W-R stars, emission line, AGBs, variables, and post-AGBs. We grouped this with the other lists to produce two categories, evolved stars which totaled 117 sources, and PNs which total 82. The sources are identified in the last column of Table 2.

\subsubsection{Estimating Contamination}

Our color-magnitude selections overlap slightly with both the red tail of the AGB stars and the luminous tail of the galaxies. Therefore we expect some contamination from these populations and the previous section confirmed this. We also expect contamination from PNs since they overlap with YSOs in color-magnitude space. How much contamination from previously unidentified members of these populations can we expect?

IR-bright PNs have double-peaked SEDs, with one peak in the optical and one in the IR and bright nebular emission lines in the optical spectrum (Kwok 1993; van der Veen et al. 1989). These have low extinction and are optically identified by definition (Reid \& Parker 2006; Leisy et al. 1997); therefore, they likely have all already been identified in optical surveys. The more evolved, low-luminosity PNs could have been missed by previous surveys but these likely overlap with galaxies in the IR color-magnitude plots so would not make it into our YSO list.

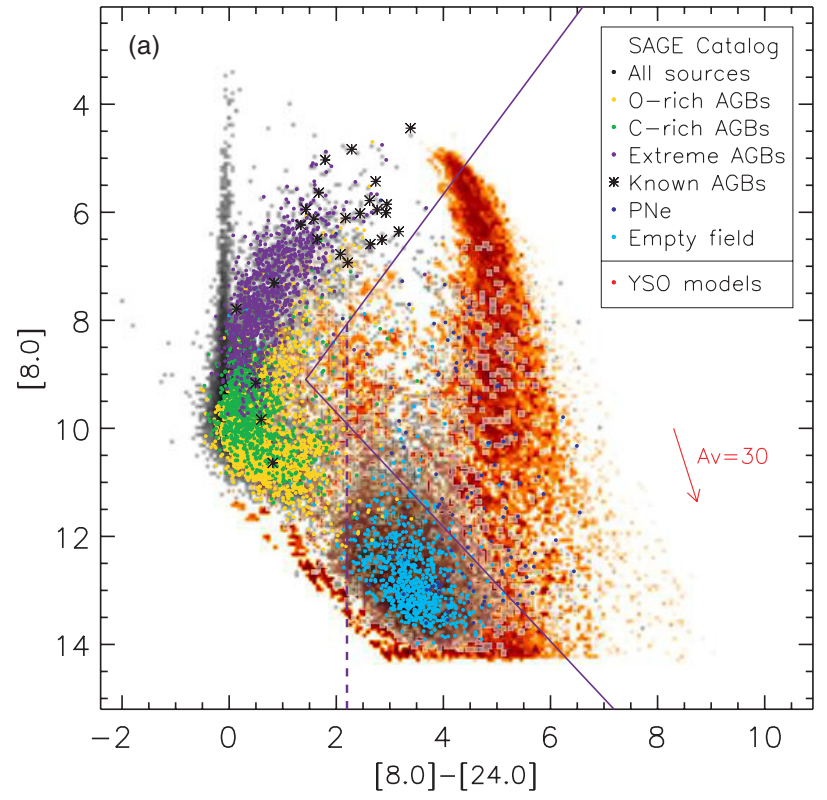

Figure 3. CMDs showing the distributions of different populations in different colors. The Catalog sources are displayed in gray scale, and the YSO models in orange scale. Subsets of the catalog from known populations are overplotted in different colors, indicated in the key. The purple lines show the boundaries between regions occupied more densely by non-YSOs with those occupied by suspected YSOs. To the right of these lines, or inside the box in some cases, are the regions from which our YSO candidate lists are selected. The interstellar reddening vectors are calculated using the "LMC average" size distribution of Weingartner \& Draine (2001). The dashed lines in Figures 3(a) and (d) denote a more stringent cut to remove AGB stars, and galaxies, respectively.

A potential source of contamination may come from proto-PNs, which have single-peaked IR-bright SEDs. We can estimate the number of proto-PNs based on the ratio of their lifetimes to the IR-bright PN stage, which is approximately 1:4 (Schoenberner 1981, 1983; Volk 1992). Thus the potential contamination from previously unidentified proto-PNs is about $1 / 4$ the number of IR-bright PNs or about $2 \%$ of the total YSO candidates.

Porras et al. (2007) used the following selection to remove galaxies from their YSO list in the Spitzer c2d survey:

$$
[8.0]>14-([4.5]-[8.0]) .
$$



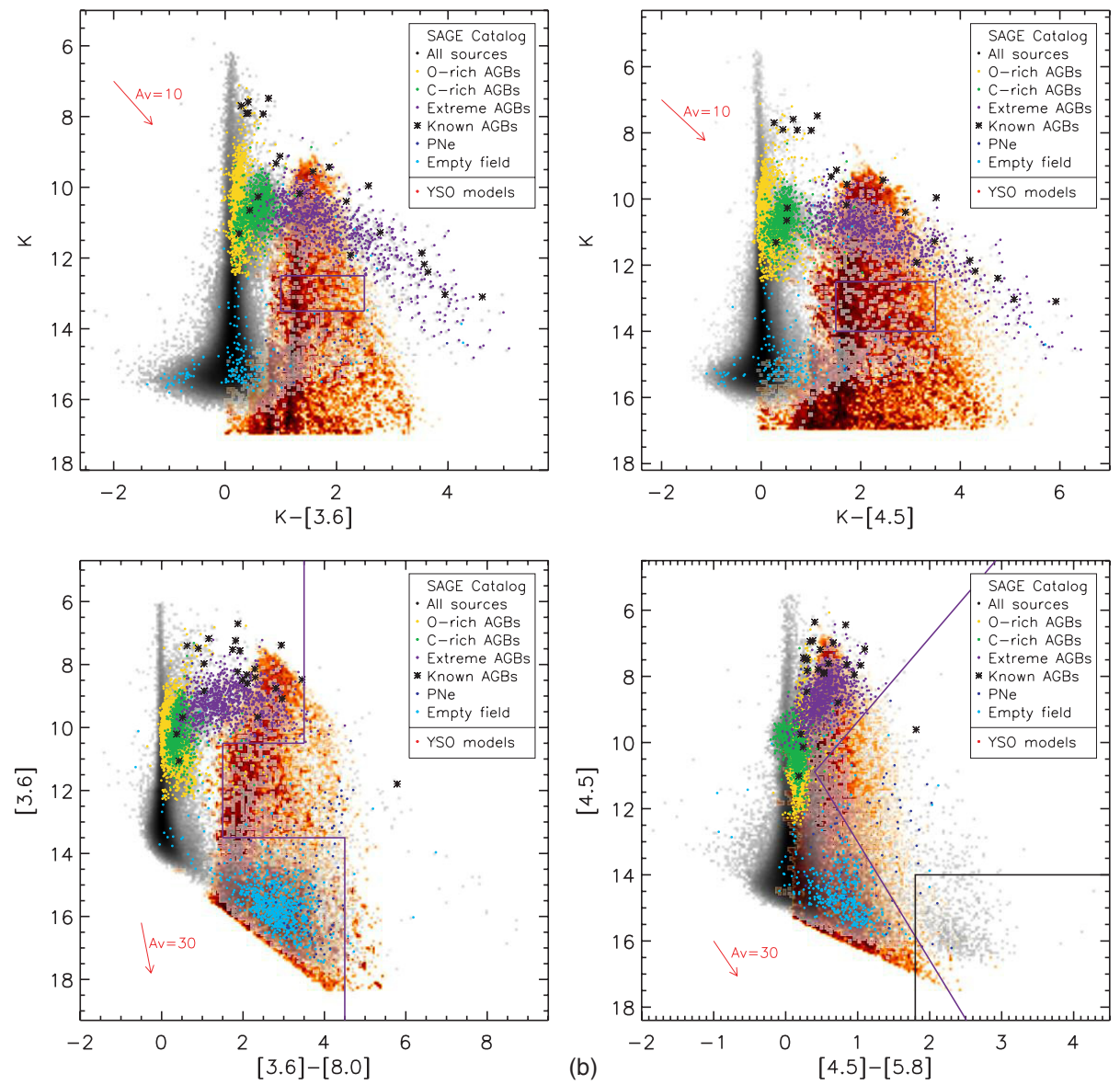

Figure 3. (Continued)

This line is shown (dashed) in the bottom right panel of Figure 3(d), which is similar to the cut we used (solid). However, our selection was logically "OR-ed" with other selections; so a source that passed another selection could have [8.0] and [4.5] magnitudes that place it below this line. The line in Equation (2) is also shown in the top-left panel of Figure 4 which plots our candidate YSO list, along with other known YSO populations; $33 \%$ of our sources fall below this line. In principle, none of these sources should be galaxies since that region of colormagnitude space was avoided in all of our color-magnitude selections. However, as stated before, the bright tail of the galaxy region did likely creep in to some of the sequences, so some of these sources are probably galaxies. To estimate a conservative upper limit for galaxy contamination, we could assume that all $33 \%$ of the sources below the Porras et al. (2007) line are galaxies.

We can do a similar conservative cut to estimate an upper limit for evolved star (AGB) contamination. Figure 3 shows that the best separation in color-space between known evolved stars (excluding PNs) and YSOs (indicated by the models) occurs in the IRAC-[24] colors (as opposed to the 2MASS-IRAC or IRAC-IRAC colors). So to remove more evolved stars, we could apply the following selection:

$$
[8.0]-[24]>2.2 \text { and }[8.0]>11-1.33([8.0]-[24]) \text {. }
$$

This is shown as the dashed line and the upper solid line in Figure 3(a). $32 \%$ of our YSO candidates fall to the left of these lines.
Some of these culled "AGB" sources overlap with the culled "galaxy" sources, so to determine the union of these sets we perform a logical "AND" of Equations (2) and (3) which removes 541 sources, or $53 \%$. Thus, we could estimate an upper limit to the contaminants in our list of about $55 \%$ including the PNs. Follow-up spectroscopy programs are already planned by several groups, which will better determine the percentage contamination and improve our ability to separate these populations in color-magnitude space.

\subsubsection{The Final YSO List}

Tables 1 and 2 show the entire list of 1197 YSO candidates. We include the 207 sources identified as non-YSOs in this table, with notations in the last column of Table 2 . We include them for completeness since not all are certain identifications. In addition, their SEDs can be instructive.

We show color-magnitude and color-color plots of the candidate YSOs in Figure 4. Overlayed on these plots are known YSOs from the literature, which will be discussed more in Section 3.1.

\section{INITIAL ANALYSIS}

\subsection{Comparison With Known YSOs}

It is interesting to compare our YSO sample with previously identified YSOs. We found four major categories of candidate LMC YSOs in the literature prior to Spitzer: IR-classified objects in a few well-studied regions, IR objects associated with masers, 

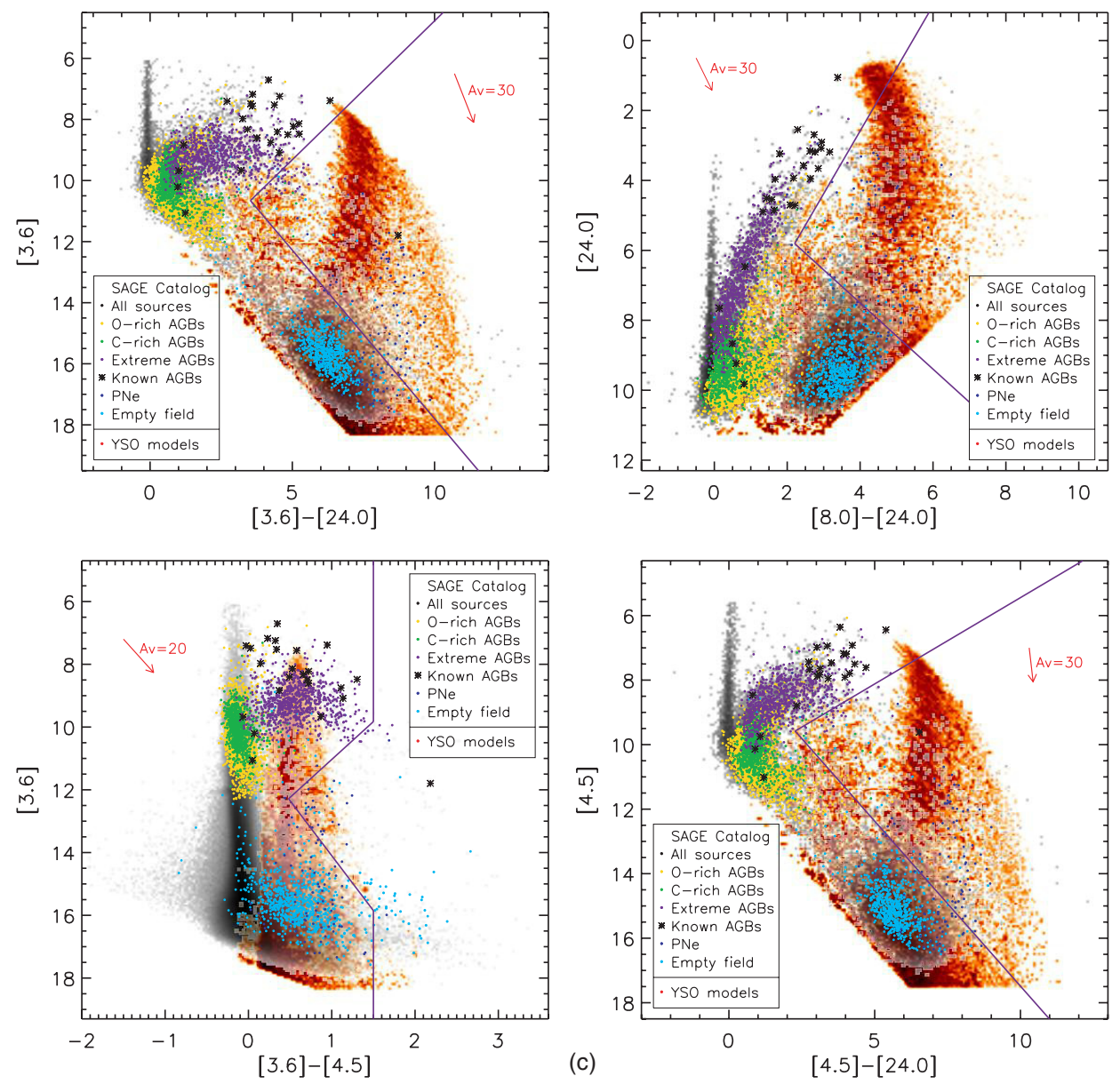

Figure 3. (Continued)

pre-MS stars identified with $H S T$ imaging, and candidate Herbig $\mathrm{Ae} / \mathrm{Be}$ stars identified by variability. Except for the numerous $H S T$-identified candidates, many of which have no IR excesses, the sources we have identified from the literature are shown in Table 3 and their magnitudes are in Table 4.

Two of the most well-studied regions in the LMC are 30 Doradus (N157), and the N159/N160 region just south of it. Brandner et al. (2001) present NICMOS near-IR imaging in the filaments near R136 in the 30 Doradus region, mostly pointed at previously identified candidate protostellar objects and knots of star formation (Hyland et al. 1992; Rubio et al. 1992, among others). They identify 24 candidate protostars, most of which are not detected in our SAGE Catalog due to the bright diffuse emission in this region; but we have identified and measured mid-IR fluxes for two sources, 30 Dor-NIC15a,b and 30DorNIC16a, in Table 4. Interestingly, each of these is resolved into a pair of protostellar candidates with NICMOS. Jones et al. (2005) present IRAC observations of N159/N160 including MIR photometry of four candidate YSOs, two of which (P1 and P2) had been previously identified in the near-IR by the same group (P1, Gatley et al. 1981; P2, Jones et al. 1986). The lower diffuse emission in this region compared to 30 Doradus allows us to estimate fluxes from SAGE data for several sources. Our fluxes agree within uncertainty with Jones et al. (2005), and we add MIPS longer-wavelength measurements in Table 4. Testor et al. (2006) present near-IR spectroscopy and photometry of the N159 region at high spatial resolution with the VLT, resolve one Jones et al. (2005) protostar (P2, here N159A7) into two, and identify another small cluster (N159A6) including a YSO candidate (N159A6-151). Finally, Chu et al. (2005) identify three candidate YSOs by their IRAC colors in dust globules in the superbubble N51D (N51D YSO-1,2, \& 3 in Tables $3 \& 4$ ).

In Galactic studies, water and methanol masers are considered to be signposts of star formation. These usually do not unambiguously identify YSOs, but can guide other searches. The LMC surveys are somewhat inconclusive, but several agree that there is maser emission associated with N160A and the SE edge of N105 (Scalise \& Braz 1982; Whiteoak et al. 1983; Ellingsen et al. 1994). Epchtein et al. (1984) identify IR counterparts to the N105 water and OH masers and one in N160A. These are included in Tables 3 and 4.

Several groups have located pre-MS stars in the LMC using HST images (Romaniello et al. 2006; Gouliermis et al. 2006; Panagia et al. 2000), by identifying objects redward of the MS in optical CMDs. These papers generally do not give position catalogs for their numerous candidates, and with Spitzer's poorer resolution, few are easily identified in the crowded starformation regions. In addition, these studies are sensitive to lowmass pre-MS stars, many of which have no circumstellar disks. Our YSO list is selected based on a mid-IR excess produced by circumstellar dust, and therefore is not expected to overlap substantially with these objects.

Finally, the EROS group, specifically de Wit and collaborators, have identified several candidate Herbig Ae/Be stars based 

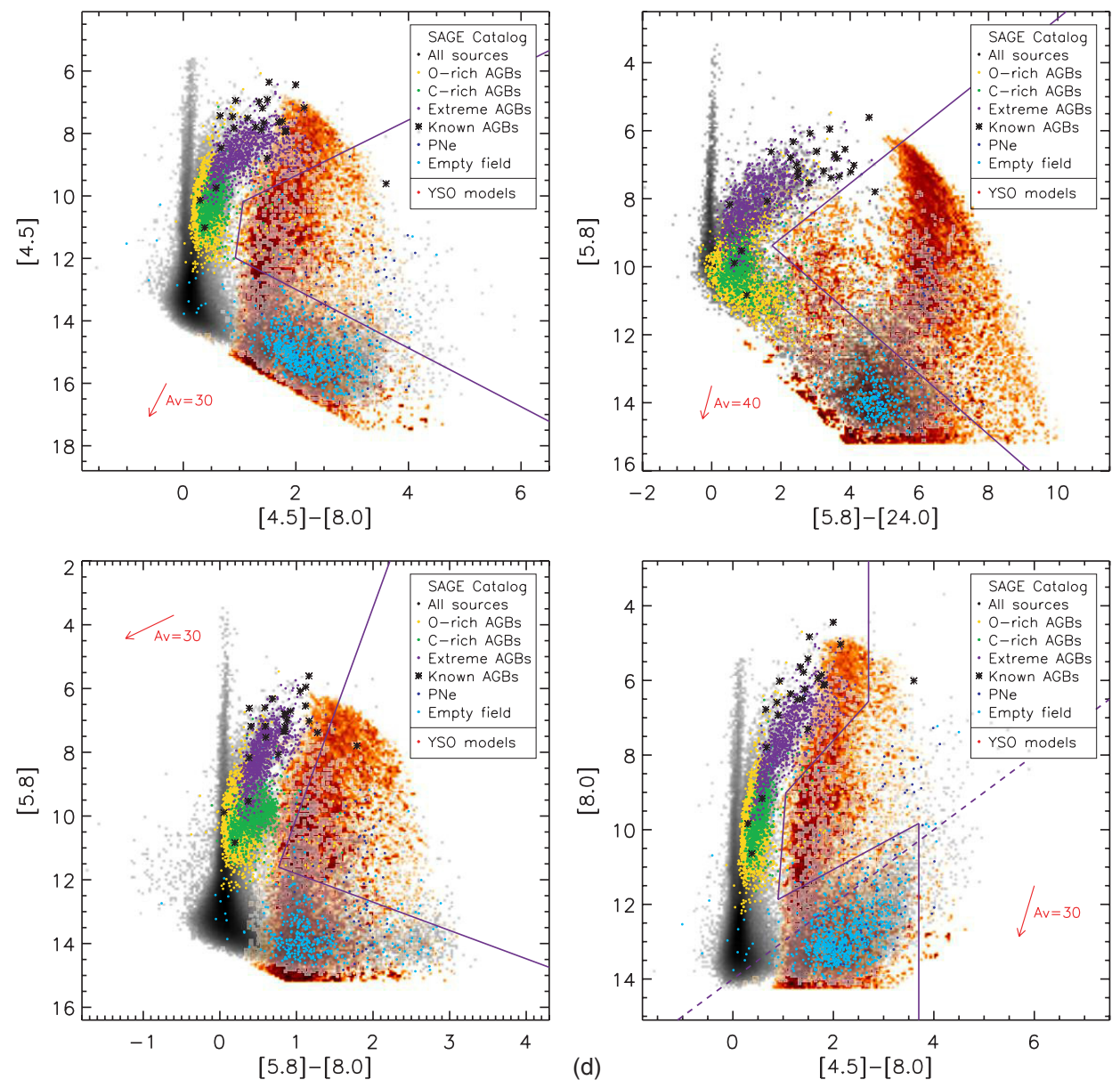

Figure 3. (Continued)

initially on optical variability, and followed up with spectra and near-IR imaging (Lamers et al. 1999; de Wit et al. 2002). Many in their sample are consistent with classical (post-MS) Be stars, but de Wit et al. (2005) identify a subset of their sample that could be pre-MS objects. Of these, three sources (ELHC 7, ELHC 13, and ELHC 19) are in the SAGE Catalog (Table 3), but not our YSO list.

Figure 4 shows that the known LMC YSOs (in red) have similar colors and are generally brighter than our YSO candidates (black). Of the 18 known sources listed in Table 3 and 4, 15 are in our SAGE Catalog. One of the remaining sources is not a point source (30Dor-NIC15a \& b), one is below our sensitivity limits (LTS J054427-692659), and the third, N51D YSO-2, is in a crowded region (sources within $0.5^{\prime \prime}$ of one another are removed from our IRAC Catalog due to photometric errors that result in crowded regions). Six of the 18 known sources are in our list of YSO candidates: N105A IRS1, N51D-YSO1, N51D-YSO3, N159-No.9, N160A, and IRAS05328-6827. Of the remaining, N159A6-151 has an IRAC-MIPS distance of $\sim 2$ "and therefore was culled from the list. Seven sources did not fulfil our color-magnitude criteria, demonstrating that our YSO list is incomplete. ELHC13 and ELHC19 do not satisfy our requirement of having at least three detections in IRAC and MIPS.

Also overlayed in Figure 4 are cataloged YSOs in the M16 region (Indebetouw et al. 2007), Galactic Ultra-Compact H II regions (Giveon et al. 2007), a Galactic H II region template (Cohen et al. 2007), and LMC compact H II regions (Buchanan et al. 2006). These have similar colors as the LMC candidate YSOs. The M16 sources have had their fluxes scaled by $(2.15 / 50)^{2}$ to place them at the same distance as the LMC. This demonstrates that the M16 sources are a lower-mass population than the LMC candidate list. The lower-mass LMC sources have been missed due to their overlap with background galaxies (Figure 3).

\subsection{Spatial Distribution and Comparison to Gas Tracers}

Figure 5 shows the YSO candidate list overlayed on the IRAC $8 \mu \mathrm{m}$ image. The YSOs appear to be spatially correlated with the brighter $8 \mu \mathrm{m}$ diffuse emission. Figure 6 shows the same as Figure 5 but zoomed in, and with massive MS stars overlayed in red. These stars have a mass greater than $10 M_{\odot}$ and ages less than 5-10 Myr. They were selected from the MCPS Catalog (Zaritsky et al. 2004) using the following criteria: $V<16$ and $B-V<0.5$ and $V<15.2+3.2(B-V)$, where $Q$ is the reddening-free parameter defined by $Q=$ $(U-B)-0.72(B-V)$. The massive MS stars tend to cluster inside the bubbles and the YSOs lie in the shells formed by the bubbles. This is consistent with both triggering in the compressed shells formed by the previous generation of massive stars (Oey \& Massey 1995; Efremov \& Elmegreen 1998b), as well as the stochastic self-propagating star-formation scenario due to differential rotation of the galaxy (Feitzinger et al. 1981). Figure 7 shows a three-color IRAC+MIPS zoom of the N11 region, with the YSOs overlayed. The lack of YSOs in the 

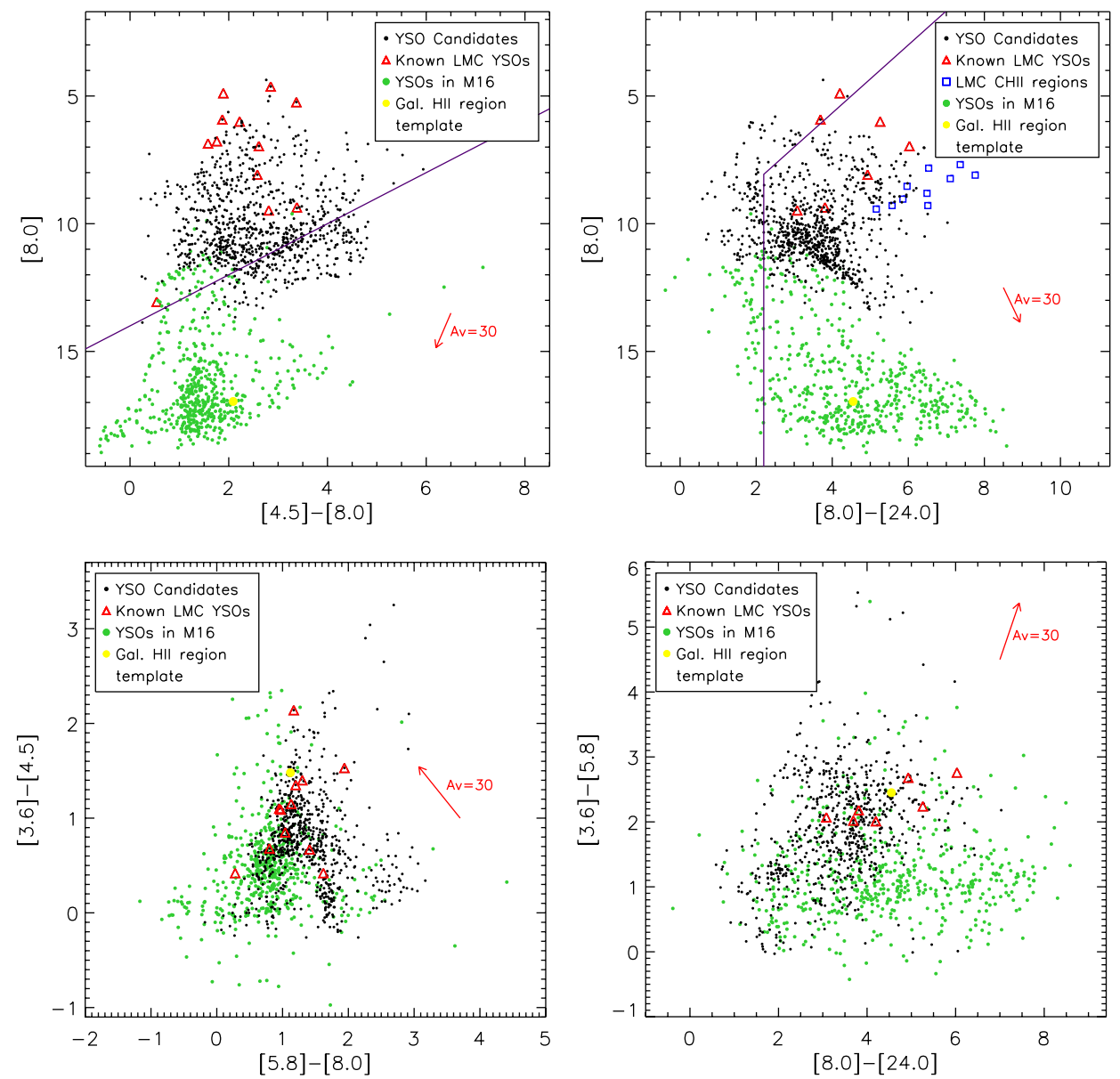

Figure 4. Color-color and color-magnitude plots of our candidate YSO list in black, with known sources overplotted in colors indicated by the key and described in Section 3.1. The purple lines in the top two panels show more stringent cuts we could made on the final list to remove galaxies (top left panel) and AGB stars (top right).

central region suggests that our list is incomplete due to bright backgrounds. Also, extended sources at IRAC bands are missing from our Point Source Catalog (for example, the string of four sources on the far-eastern side of the image).

Figure 8 shows the YSOs overlayed on a two-color image of H I gas (Staveley-Smith et al. 2003) in green and CO gas (Fukui et al. 1999) in red. The YSOs appear to be associated with both the neutral and molecular gas, with a preference for the molecular gas (yellow and red). This will be quantified shortly with correlation plots. The southeast ridge and arc at lower left are prominent in the CO (in red and yellow). This is the leading edge of the LMC in its motion through the halo of the Milky Way. de Boer et al. (1998) proposed that star formation is triggered in this compressed region due to the bowshock of the LMC. As the galaxy rotates clockwise, material moves away (to the north), and we should find a progression in the ages toward the north. To the north lies in succession N159, 30 Doradus, and in the far north, the large LMC 4 shell, each successively older, so the picture qualitatively fits. We do see active star formation in the southeast ridge, as indicated by the number of YSOs in Figures 5 and 8. In fact, Indebetouw (2008) find an SFR in this region of $0.14 M_{\odot} \mathrm{yr}^{-1} \mathrm{kpc}^{-2}$, which is a few times higher than the global rate per area estimated in this paper (Section 3.5).

Figures 5 and 8 show that the YSO candidates are spatially correlated with the gas in the galaxy. This can be quantified in several ways. First, we can calculate the correlation coefficients between the spatial density of YSOs and the $\mathrm{CO}$ and $\mathrm{H}$ i column densities. The spatial density of candidates can be calculated robustly at 10 arcmin resolution (interpretation at finer spatial resolution is difficult with only about 40 sources per square degree), and the $\mathrm{CO}$ and $\mathrm{H}$ I maps were smoothed to the same resolution. This gives correlation coefficients of $0.82 \pm 0.03$ and $0.73 \pm 0.05$, for the $\mathrm{CO}$ and $\mathrm{H}_{\mathrm{I}}$ maps, respectively. The uncertainty quoted is the spread in the result using different smoothing kernels and using either a fixed grid or density-scaled grid method to construct the source density map.

Another measure of gas-YSO correlation is the distribution of gas column density at the location of the YSOs compared to the distribution for the general Point Source Catalog. Figures 9-11 shows these distributions for $\mathrm{H}$, $\mathrm{CO}, 24 \mu \mathrm{m}$, and $8 \mu \mathrm{m}$ diffuse emissions. Since the YSO list was initially culled if the diffuse $24 \mu \mathrm{m}$ emission was greater than $0.08 \mathrm{MJy} \mathrm{sr}^{-1}$, we applied the same culling to the general catalog in these plots. Then if the YSOs show more correlation, it is due to a real spatial association. These figures show that YSO candidates are statistically associated with greater amounts of gas than generic point sources in the LMC. The peak of the column density distribution associated with YSOs is $\sim 2 \times 10^{21} \mathrm{~cm}^{-2}$ of atomic hydrogen (Figure 9, left). This is consistent with the threshold for the formation of molecular material (approximately 
Table 3

Previously Known YSOs

\begin{tabular}{|c|c|c|c|c|c|}
\hline Source & $\begin{array}{l}\text { R.A. (J2000) } \\
(\mathrm{h} \mathrm{m} \mathrm{s})\end{array}$ & $\begin{array}{c}\text { Decl. }(\mathrm{J} 2000) \\
\left({ }^{\circ} \prime \prime \prime\right)\end{array}$ & IRAC designation & $\begin{array}{c}\text { Separation } \\
\text { IRAC/MIPS } \\
(\operatorname{arcsec})\end{array}$ & Ref. \\
\hline 30Dor-NIC15a. . & 053848.30 & -690410.3 & $\cdots$ & $\cdots$ & 1 \\
\hline 30Dor-NIC15b... & 053848.45 & -690412.0 & $\cdots$ & $\cdots$ & 1 \\
\hline 30Dor-NIC16a... & 053841.62 & -690354.7 & SSTISAGE1C J053841.36-690354.2 & 1.5 & 1 \\
\hline N159-P1... & 053959.36 & -694526.3 & SSTISAGE1C J053959.30-694526.2 & 0.3 & 2 \\
\hline $\mathrm{N} 159-\mathrm{P} 2{ }^{\mathrm{a}} \ldots$ & 053941.88 & -694612.2 & SSTISAGE1C J053941.85-694611.9 & 0.3 & 2 \\
\hline N159-No.9. . . & 053937.12 & -694537.0 & SSTISAGE1C J053937.01-694536.7 & 0.6 & 2 \\
\hline N159-No.134. . . & 054019.12 & -694445.6 & SSTISAGE1C J054018.97-694445.5 & 0.8 & 2 \\
\hline N159A6-151 ${ }^{\mathrm{b}} \ldots$ & 053936.17 & -694604.3 & SSTISAGE1C J053935.97-694604.0 & $1.1 / 1.0$ & 3 \\
\hline N160A... & 053943.66 & -693830.2 & SSTISAGE1C J053943.82-693833.8 & 3.8 & 4 \\
\hline IRAS05328-6827. . . & 053238.59 & -682522.2 & SSTISAGE1C J053238.58-682522.3 & $0.2 / 0.6$ & 5 \\
\hline N157B IRS1... & 053750.3 & -691107 & SSTISAGE1C J053750.28-691107.1 & 0.1 & 6 \\
\hline N105A IRS1... & 050950.6 & -685305 & SSTISAGE1C J050950.53-685305.4 & 0.6 & 6 \\
\hline N51D YSO-1... & 052601.30 & -673011.8 & SSTISAGE1C J052601.22-673011.8 & $0.5 / 0.6$ & 7 \\
\hline N51D YSO-2... & 052604.01 & -672957.0 & $\cdots$ & $\cdots$ & 7 \\
\hline N51D YSO-3... & 052619.91 & -673033.3 & SSTISAGE1C J052619.79-673033.3 & $0.7 / 0.9$ & 7 \\
\hline LTS J054427-692659 $\ldots$ & 054427.47 & -692659.2 & $\cdots$ & $\cdots$ & 8 \\
\hline $\mathrm{ELHC7}^{\mathrm{d}}$. & 051639.5 & -692049 & SSTISAGE1C J051639.18-692048.1 & 1.9 & 9 \\
\hline ELHC13 $\ldots$ & 051854.91 & -693635.8 & SSTISAGE1C J051854.69-693635.5 & $1.1 / 1.4$ & 9 \\
\hline ELHC19 ${ }^{\mathrm{e}} \ldots$ & 051711.80 & -692554.0 & SSTISAGE1C J051711.59-692555.3 & 1.8 & 9 \\
\hline
\end{tabular}

Notes.

${ }^{\mathrm{a}} \mathrm{P} 2$ was resolved into two components by Testor et al. (2006, resolution $\left.0^{\text {“ }} .2\right)$ : N159A7-121 $\left(\alpha=05^{\mathrm{h}} 39^{\mathrm{m}} 41.96^{\mathrm{s}}\right.$ and $\left.\delta=-69^{\circ} 46^{\prime} 11.99^{\prime \prime}, \mathrm{J} 2000\right)$ and N159A7-123 $\left(\alpha=05^{\mathrm{h}} 39^{\mathrm{m}} 41.90^{\mathrm{s}}\right.$ and $\left.\delta=-69^{\circ} 46^{\prime} 11.52^{\prime \prime}, \mathrm{J} 2000\right)$, both classified as Class I YSOs.

b The compact source N159A6 discovered by Testor et al. (2006) consists of two objects contained within a diameter of 0.5 pc: an YSO N159A6-151 and a CHII/HII region containing about five stars. At the IRAC resolution of $2^{\prime \prime}$ $(0.48 \mathrm{pc}$ at $50 \mathrm{kpc}), \mathrm{N} 159 \mathrm{~A} 6$ will be either unresolved or only slightly resolved.

c LTS ("LMC T Tauri Star") J054427-692659 is the first spectroscopically confirmed discovery of T Tauri star in the LMC.

${ }^{\mathrm{d}}$ ELHC stands for EROS LMC HAe/Be Candidates Lamers et al. (1999). ELHC7 is a confirmed HAe/Be UXOrionis star.

e de Wit et al. (2005) suggested that the type of variability of these sources could be interpreted as caused by variable dust obscuration; thus it is possible that they are pre-MS objects. However, these sources lack thermal dust emission in the near IR. The circumstellar dust emission could be revealed in the mid- or far IR; thus the observations at these wavelengths are crucial to confirm that these objects are in a pre-MS stage.

References.

(1) Brandner et al. (2001); (2) Jones et al. (2005); (3) Testor et al. (2006); (4) Epchtein et al. (1984); (5) van Loon et al. (2005); (6) Oliveira et al. (2006); (7) Chu et al. (2005); (8) Wichmann et al. (2001); (9) de Wit et al. (2005), and references therein.

$10^{21} \mathrm{~cm}^{-2}$, somewhat higher with the porous geometry, lower metallicity, and lower dust-to-gas ratio). On the other hand, this value may only be coincidental, since the column density associated with generic catalog sources peaks only about a factor of 2 lower, because the typical value of the Hi column density in the galaxy is $10^{21} \mathrm{~cm}^{-2}$. Additionally, in many places along the line of sight there are (at least) two kinematically and likely physically distinct gas components (Luks \& Rohlfs 1992; Mizuno et al. 2001) so the relevant column for self-shielding may be less than half of the total along the line of sight.

The right panel of Figure 9 shows the relative distributions of YSOs and point sources as a function of peak $\mathrm{H}$ i intensity rather than the total column. One might expect the peak $\mathrm{H}$ i to better reflect the densest individual clouds along the sightline, and correlate better with star formation. Indeed, the distributions are rather more separated than for column density.

The relative distributions as a function of $\mathrm{CO}$ column density (Figure 10) are similar to those for $\mathrm{HI}$, with a shift at a few $10^{20} \mathrm{~cm}^{-2}$, when using a Galactic value of the X-factor (for converting $\mathrm{CO}$ column density to $\mathrm{H}_{2}$ ), $3 \times 10^{20} \mathrm{~cm}^{-2}$ $\left(\mathrm{K} \mathrm{km} \mathrm{s}^{-1}\right)^{-1}$. If we adopt a higher value of the $\mathrm{X}$-factor, as suggested by various studies including the NANTEN survey (Fukui et al. 1999), the characteristic value is approximately $10^{21} \mathrm{~cm}^{-2}$. As expected, there is a stronger association with dense $\mathrm{CO}$ gas than $\mathrm{H}$ i gas.

Figure 11 shows the spatial association of YSOs with $24 \mu \mathrm{m}$ and $8 \mu \mathrm{m}$ surface brightness. At first glance, it appears that the YSOs are more strongly associated with higher levels of $8 \mu \mathrm{m}$ flux than $24 \mu \mathrm{m}$ flux. However, the YSO distributions themselves, shown as hatched regions, are fairly similar in the two panels. It is the SAGE Catalog, in gray, that is different, associated with low values of $8 \mu \mathrm{m}$ sky and with a range of $24 \mu \mathrm{m}$ sky values. The SAGE Catalog consists mostly of stars detected in the 3.6 and $4.5 \mu \mathrm{m}$ bands. As Figure 6 shows, the massive stars are located preferentially inside the $8 \mu \mathrm{m}$ shells and $\mathrm{H}_{\mathrm{II}}$ regions. A similar effect is seen in the SMC where the regions surrounding ionizing sources are mostly devoid of $8 \mu \mathrm{m}$ emission (Bolatto et al. 2007). The $24 \mu \mathrm{m}$ diffuse emission is bright in the shells but also bright inside the $\mathrm{H}_{\text {II }}$ regions where massive stars are found, as seen in Figure 4 of Meixner et al. (2006) and in Figure 7 of this paper. Thus, the massive stars are spatially anti-correlated with $8 \mu \mathrm{m}$ emission and associated 
Table 4

Previously Known YSOs: Magnitudes ${ }^{\mathrm{a}}$

\begin{tabular}{|c|c|c|c|c|c|c|c|c|c|}
\hline \multirow[t]{2}{*}{ Source } & \multicolumn{3}{|c|}{ 2MASS } & \multicolumn{4}{|c|}{ IRAC } & \multirow{2}{*}{$\begin{array}{c}\text { MIPS } \\
{[24]} \\
\end{array}$} & \multirow[t]{2}{*}{ Ref. } \\
\hline & $J$ & $H$ & $K_{s}$ & [3.6] & [4.5] & [5.8] & [8.0] & & \\
\hline 30Dor-NIC15a... & $19.01(0.08)^{\mathrm{b}}$ & $18.24(0.13)^{\mathrm{b}}$ & $17.57(0.16)^{b}$ & & & & & & 1 \\
\hline 30Dor-NIC15b... & $17.06(0.07)^{\mathrm{b}}$ & $15.25(0.11)^{b}$ & $13.49(0.12)^{\mathrm{b}}$ & $9.24(0.04)^{\mathrm{c}}$ & $8.53(0.04)^{\mathrm{c}}$ & $6.76(0.04)^{\mathrm{c}}$ & $4.97(0.04)^{\mathrm{c}}$ & $<0.61(0.11)^{\mathrm{d}}$ & 1 \\
\hline 30Dor-NIC16a... & $15.74(0.08)$ & $13.31(0.02)$ & $11.51(0.02)$ & $9.12(0.06)$ & $8.44(0.09)$ & $7.66(0.03)$ & $6.86(0.03)$ & $\ldots$ & 1 \\
\hline N159-P1... & $16.46(0.17)$ & $13.97(0.08)$ & $11.84(0.03)$ & $9.38(0.07)$ & $8.53(0.07)$ & $7.81(0.04)$ & $6.77(0.05)$ & $\ldots$ & 2 \\
\hline N159-P2. . & $15.29(0.19)$ & $14.04(0.15)$ & $12.16(0.05)$ & $9.37(0.09)$ & $8.22(0.07)$ & $7.13(0.04)$ & $6.00(0.08)$ & $0.74(0.01)^{\mathrm{c}}$ & 2 \\
\hline N159-No.9. . & $15.55^{\mathrm{b}}$ & $14.25^{\mathrm{b}}$ & $14.23(0.12)$ & $10.92(0.09)$ & $9.57(0.07)$ & $8.16(0.06)$ & $6.96(0.10)$ & $0.93(0.01)^{\mathrm{c}}$ & 2 \\
\hline N159-No.134. . & & $\ldots$ & & $14.28(0.11)$ & $12.89(0.14)$ & $11.58(0.10)$ & $\ldots$ & $\ldots$ & 2 \\
\hline N159A6-151... & $17.91^{\mathrm{b}}$ & $\ldots$ & $14.47^{\mathrm{b}}$ & $11.24(0.12)$ & $\ldots$ & $9.02(0.11)$ & $\ldots$ & $2.00(0.02)$ & 3 \\
\hline N160A... & $14.17(0.21)$ & $14.04(0.38)$ & $12.90(0.15)$ & $9.62(0.09)$ & $7.48(0.05)$ & $5.80(0.03)$ & $4.63(0.04)$ & $\ldots$ & 4 \\
\hline IRAS05328-6827. . & $16.65(0.20)$ & $14.24(0.06)$ & $11.98(0.03)$ & $8.89(0.05)$ & $7.79(0.05)$ & $6.87(0.02)$ & $5.92(0.02)$ & $2.23(0.01)$ & 5 \\
\hline N157B IRS1... & $15.93(0.12)^{\mathrm{b}}$ & $14.22(0.07)$ & $11.45(0.03)$ & $7.87(0.04)$ & $6.78(0.03)$ & $5.86(0.03)$ & $4.89(0.02)$ & $0.69(0.01)^{\mathrm{c}}$ & 6 \\
\hline N105A IRS1... & $>15.3^{\mathrm{b}}$ & $>14.7^{\mathrm{b}}$ & $13.77(0.10)$ & $10.14(0.04)$ & $8.61(0.04)$ & $7.18(0.02)$ & $5.24(0.02)$ & $\ldots$ & 6 \\
\hline N51D YSO-1... & $\ldots$ & $\ldots$ & $\ldots$ & $12.06(1.21)^{\mathrm{b}}$ & $10.66(0.08)$ & $9.38(0.06)$ & $8.08(0.06)$ & $3.15(0.01)$ & 7 \\
\hline N51D YSO-2... & $\ldots$ & $\ldots$ & $\ldots$ & $13.16(1.32)^{\mathrm{b}}$ & $12.74(1.27)^{\mathrm{b}}$ & $10.98(1.10)^{\mathrm{b}}$ & $9.36(0.94)^{b}$ & $5.55(0.56)^{\mathrm{b}}$ & 7 \\
\hline N51D YSO-3... & $\ldots$ & $\ldots$ & $14.95(0.13)$ & $12.96(0.1)$ & $12.29(0.08)$ & $10.89(0.05)$ & $9.48(0.07)$ & $6.40(0.01)$ & 7 \\
\hline LTS J054427-692659. . . & $20.73(0.09)^{\mathrm{b}}$ & $19.45(0.04)^{\mathrm{b}}$ & $18.57(0.06)^{\mathrm{b}}$ & $\ldots$ & $\ldots$ & $\ldots$ & $\ldots$ & $\ldots$ & 8 \\
\hline ELHC7... & $17.60^{\mathrm{b}}$ & $16.99^{\mathrm{b}}$ & $15.96^{\mathrm{b}}$ & $14.02(0.04)$ & $13.60(0.06)$ & $13.34(0.08)$ & $13.06(0.09)$ & $\ldots$ & 9 \\
\hline ELHC13... & $\ldots$ & $\ldots$ & $\ldots$ & $\ldots$ & $14.51(0.12)$ & $\ldots$ & $\ldots$ & $8.10(0.05)$ & 9 \\
\hline ELHC19... & $16.35^{\mathrm{b}}$ & $16.23^{\mathrm{b}}$ & $16.10^{\mathrm{b}}$ & $16.05(0.11)$ & $16.09(0.14)$ & $\ldots$ & $\ldots$ & $\ldots$ & 9 \\
\hline
\end{tabular}

Notes.

${ }^{a}$ Magnitudes from the SAGE Catalog are given where available.

b Data from literature (see references below).

${ }^{\mathrm{c}}$ Magnitudes measured in this work using aperture photometry.

d Source saturated at $24 \mu \mathrm{m}$.

References.

(1) Brandner et al. (2001); (2) Jones et al. (2005); (3) Testor et al. (2006); (4) Epchtein et al. (1984); (5) van Loon et al. (2005); (6) Oliveira et al. (2006); (7) Chu et al. (2005); (8) Wichmann et al. (2001); (9) de Wit et al. (2005), and references therein.

with $24 \mu \mathrm{m}$ emission in HiI regions, whereas the YSOs are associated with high $8 \mu \mathrm{m}$ emission. This is another way of showing the visual result from Figure 6 that star formation takes place in the shells created by the previous generation of highmass star formation (the massive stars inside the shells).

Figure 12 shows the two-point correlation function of YSO candidates compared to generic point sources. The generic point sources (dot-dashed) are not clustered, but the YSOs (dashed) are highly clustered, as expected if star formation takes place in filamentary gas clouds. The YSOs in H I clouds (solid sources associated with $>30 \mathrm{~K} \mathrm{~km} \mathrm{~s}^{-1}$ ) are even more clustered. These data do not show any particular characteristic physical scale (as would be evident in a break in the power-law slope of the correlation function). If star formation is dominated by selfpropagation in overlapping supershells (Oey \& Massey 1995; Efremov \& Elmegreen 1998a), it should be fairly scale-free in the range of physical scales we are able to probe, but it is possible that the tail of elevated correlation at size scales of $\sim 0.1^{\circ} \simeq$ $450 \mathrm{pc}$ is associated with supershells.

Figure 12 also shows the nearest-neighbor distribution of our YSO candidates. The nearest-neighbor distribution can reflect size scales in the molecular cloud at the time of star formationfor example in the Galaxy, a signature of the cloud's Jeans length has been seen in the YSO distribution of NGC2264 (Teixeira et al. 2006) and possibly in M16 (Indebetouw et al. 2007). In the LMC we see an excess in the nearest-neighbor distribution of YSOs compared to generic point sources at the smallest size scales to which our analysis is sensitive $\left(\sim 10^{\prime \prime} \simeq 3 \mathrm{pc}\right)$. Given that we are limited by the spatial resolution at $24 \mu \mathrm{m}$, we cannot interpret this as a physical scale, but this provides further evidence that YSO candidates are highly clustered in the smallest scales accessible to Spitzer.

\subsection{Linear Regression SED Fitting to All the Sources}

We fitted YSO models to the SEDs of all of the sources in Table 1, using the model grid described in Robitaille et al. (2006) and the fitting method described in Robitaille et al. (2007). Based on recent experience fitting YSO data (Robitaille et al. 2007; Indebetouw et al. 2007; Simon et al. 2007; Shepherd et al. 2007; Watson et al. 2008) we reset the photometric errors to $10 \%$ in each band. This allows for other errors (systematic, calibration, variability) besides the photon-counting errors usually quoted. We define well-fitted models as those whose $\chi^{2}$ per data point is less than two, based on by-eye examination of the fits. In many cases, the poorly-fitted sources are due to one bad data point, mismatch between 2MASS and Spitzer due to variability, multiple sources in the beam, or inadequacies in the models. Thus a poor fit does not necessarily indicate that the source is not a YSO.

Example SEDs and YSO fits of different categories of objects are shown in Figure 13, and the rest are available in the online journal (Figures 13.1-13.80). For the list with identified nonYSO sources removed, 570/990 sources were well fitted (that is, have a $\chi^{2}$ per data point less than 2). Thus, $58 \%$ of the candidate YSOs were well fitted by YSO models. For the PNs 54/82 (65\%) were well fitted by the YSO models. The SEDs and colors of the PNs generally resemble those of the YSOs as shown in Figures 3 and 13. For the other categories, 16/117 (14\%) evolved stars were well fitted, SN87a was well fitted, 2/4 galaxies were well fitted, and 2/2 X-ray sources were well fitted. Note that a lower fraction of evolved stars were well fitted than any other category. Thus the YSO fitter can be used to identify suspected evolved stars as poorly fitted with YSO models. A common reason for the poor fits of the evolved stars are that the YSO 


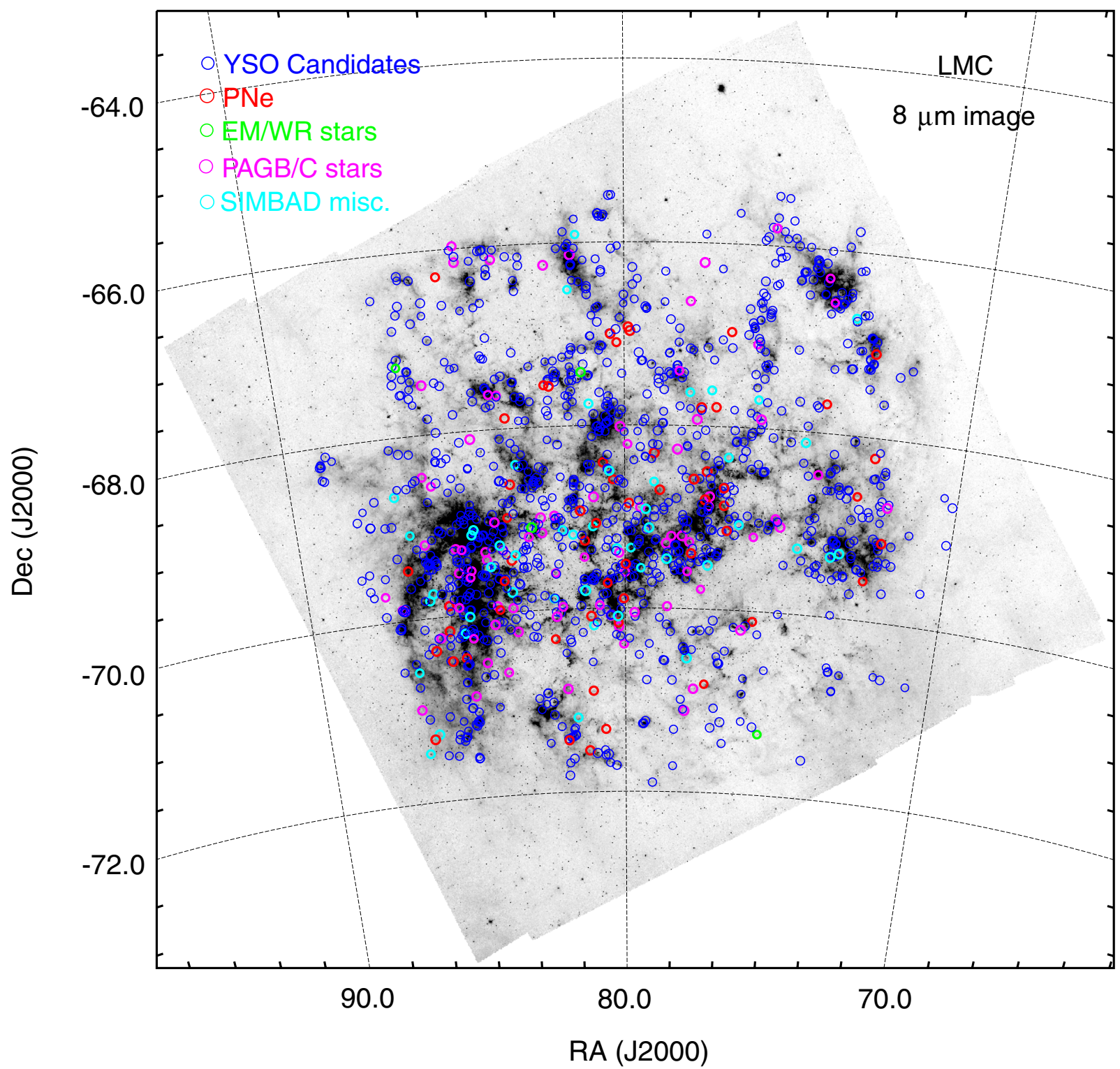

Figure 5. YSO candidates overlayed on a gray-scale $8 \mu \mathrm{m}$ image of the LMC. The sources identified as other populations (Section 2.3.3) are also shown, color coded as in the key.

models do not have the high luminosity but relatively cool photospheres needed to fit these sources. An example of this is shown in the bottom two panels of Figure 14. At the left is a YSO fit with a stellar temperature of $\sim 30,000 \mathrm{~K}$ and an IR excess from a disk. The bottom right-hand panel shows that a YSO model at a distance of only $1.5 \mathrm{kpc}$ is easily well fitted with a photosphere of $\sim 6600 \mathrm{~K}$. For these sources to be fitted with YSO models, either the pre-MS evolutionary tracks need to be modified to allow for larger stellar radii for a modest stellar temperature, or clusters of stars with average photospheric temperatures of $5000 \mathrm{~K}$ are required to fit the sources. More likely, they are evolved stars with expanded, luminous, and relatively cool photospheres.

Some examples of poorly-fitted YSOs are given in Figure 14. Based on their location in the N11 star-forming region (sources "3" and "4" in Figure 7) and their SED shapes, these are likely true YSOs. The reason the source on the left is not well fitted is that it probably has PAH emission, which is not accounted for in our models. This gives an excess at 3.6, 5.8, and especially $8 \mu \mathrm{m}$, compared to 4.5 . The source on the right has higher $J H K_{s}$ fluxes than is typical of an embedded YSO. There could be several reasons for this: variability of the source between the epochs of the 2MASS and SAGE observations; multiple sources in the beam (one optically bright and one IR bright); or incomplete sampling of model parameters in the grid. At a distance of the LMC, it would not be surprising to have more than one source in the $0.5-1 \mathrm{pc}$ IRAC beam. Also noticeable in the SEDs in Figure 13.1-13.80 are the noisier 2MASS data relative to the deeper SAGE data. This contributes several poorly-fitted SEDs as well. In addition the 2MASS resolution is $50 \%$ worse than IRAC, contributing to the confusion issue.

From these fits, we conclude that the YSO fitter can be used as an additional tool to remove contaminants since it does not fit 


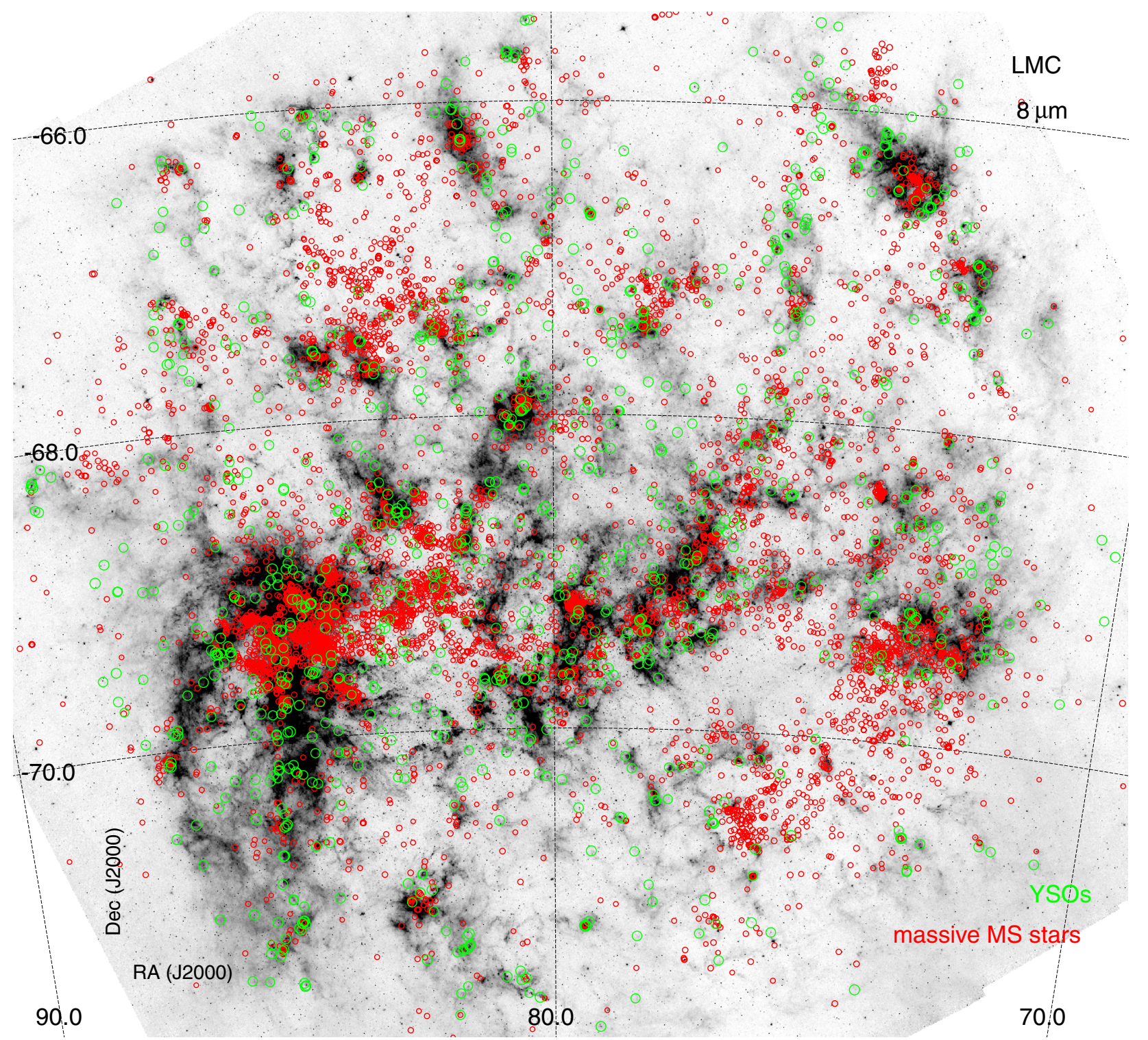

Figure 6. YSO candidates and young MS stars ( $<5-10 \mathrm{Myr})$ overlayed on a gray-scale $8 \mu \mathrm{m}$ image.

the evolved stars as well as other populations. PNs and galaxies have similar SEDs to YSOs and must be removed by other means. But it is likely that there are few undiscovered PNs in our list (Section 2.3.4). However, many of the poorly fitted YSO candidates appear to be true YSOs based on their locations in star-forming regions and SED shapes.

\subsection{Selecting a Subset of Highly Probable YSOs for Further Analysis}

We applied a further color-magnitude selection to the YSO candidate list to form a subset of more highly probable YSOs. As stated in Section 2.3.4, we can remove galaxies with the selection in Equation (2) and the AGB stars with the selection in Equation (3). These are also removing valid YSOs, but the remaining list should consist predominantly of YSOs. Applying the cut to our full list of 1197 sources, we find 559 sources. Comparing to our known contaminants we find $49 \mathrm{PNe}, 0 \mathrm{WR}$ stars, 1-emission-line stars, 0 post-AGBs, 1-carbon stars, and 18 SIMBAD stars (compared to 82, 2, 5, 47, 10, 61 respectively in the complete YSO list). This cut was most successful at removing post-AGBs and carbon stars, as expected. We removed the remaining known contaminants from the list, leaving 458 sources, denoted as "YSO_hp" in Table 2.

Fitting these sources with YSO models, we find that 299 are fitted with $\chi^{2}$ per data point less than 2 . We show some of the derived physical properties for these 299 sources in Table 5. These are the average stellar mass, luminosity, disk mass, and envelope infall rate, as well as their standard deviations. If we define $\mathrm{cpd}_{\min }$ as the best-fitted (minimum) $\chi^{2}$ per data point, all fits with cpd between $\mathrm{cpd}_{\min }$ and $\mathrm{cpd}_{\min }+1$ are used to compute the averages and standard deviations in the table. The best-fitted $\chi^{2}$ per data point is shown in Column 3 and the number of fits used in the averages is in Column 4 . The variation of fitted parameters gives an idea of how uniquely the parameters can be determined. The average ratio of the standard deviation to the fitted parameter for the 299 sources is 0.2 for mass, 0.7 for luminosity, 4 for infall rate, and 1.6 for disk mass. The derived infall rates and disk masses appear less certain than luminosity and stellar mass. However, determining the infall rate within an 
Table 5

Physical Parameters of Selected YSO Candidates

\begin{tabular}{|c|c|c|c|c|c|c|c|c|c|c|c|c|}
\hline No. & IRAC designation & $\begin{array}{c}\chi_{\min }^{2} \\
\left(\operatorname{cpd}^{\mathrm{a}}\right)\end{array}$ & $n_{\text {fits }}{ }^{b}$ & $\begin{array}{l}M_{\star}^{\text {ave }} \\
\left(M_{\odot}\right) \\
\end{array}$ & $\begin{array}{c}\text { std } \\
\left(M_{\odot}\right)\end{array}$ & $\begin{array}{l}L_{\star}^{\text {ave }} \\
\left(L_{\odot}\right) \\
\end{array}$ & $\begin{array}{c}\text { std } \\
\left(L_{\odot}\right)\end{array}$ & $\begin{array}{c}\dot{\mathrm{M}}_{\mathrm{env}}^{\text {ave }} \\
\left(M_{\odot} \mathrm{yr}^{-1}\right)\end{array}$ & $\begin{array}{c}\text { std } \\
\left(M_{\odot} \mathrm{yr}^{-1}\right)\end{array}$ & $\begin{array}{l}M_{\text {disk }}^{\text {ave }} \\
\left(M_{\odot}\right) \\
\end{array}$ & $\begin{array}{c}\text { std } \\
\left(M_{\odot}\right)\end{array}$ & $\begin{array}{l}\text { Evol. } \\
\text { stage }\end{array}$ \\
\hline 2 & SSTISAGE1CJ044037.28-690321.6 & 0.01 & 422 & 7.77 & 1.35 & $2.89 \mathrm{E}+03$ & $1.93 \mathrm{E}+03$ & $2.03 \mathrm{E}-05$ & $2.44 \mathrm{E}-04$ & $5.70 \mathrm{E}-02$ & $9.66 \mathrm{E}-02$ & I \\
\hline 4 & SSTISAGE1CJ044254.47-693719.1 & 0.01 & 305 & 7.50 & 1.27 & $2.54 \mathrm{E}+03$ & $1.67 \mathrm{E}+03$ & $3.12 \mathrm{E}-06$ & $5.44 \mathrm{E}-05$ & $2.28 \mathrm{E}-02$ & $5.45 \mathrm{E}-02$ & II \\
\hline 6 & SSTISAGE1CJ044515.41-690038.9 & 0.00 & 103 & 6.78 & 1.60 & $1.97 \mathrm{E}+03$ & $1.76 \mathrm{E}+03$ & $4.17 \mathrm{E}-08$ & $1.23 \mathrm{E}-07$ & $1.91 \mathrm{E}-02$ & $5.87 \mathrm{E}-02$ & II \\
\hline 9 & SSTISAGE1CJ044716.69-671339.2 & 1.42 & 38 & 8.74 & 3.35 & $6.07 \mathrm{E}+03$ & $1.13 \mathrm{E}+04$ & $1.73 \mathrm{E}-07$ & $3.68 \mathrm{E}-07$ & $9.69 \mathrm{E}-02$ & $1.95 \mathrm{E}-01$ & II \\
\hline 14 & SSTISAGE1CJ044808.79-684219.4 & 0.06 & 126 & 8.83 & 2.37 & $5.09 \mathrm{E}+03$ & $5.08 \mathrm{E}+03$ & $7.52 \mathrm{E}-07$ & $5.33 \mathrm{E}-06$ & $5.45 \mathrm{E}-02$ & $1.24 \mathrm{E}-01$ & II \\
\hline 18 & SSTISAGE1CJ044837.76-692337.0 & 0.11 & 33 & 16.23 & 3.53 & $2.66 \mathrm{E}+04$ & $1.75 \mathrm{E}+04$ & $4.19 \mathrm{E}-04$ & $1.65 \mathrm{E}-03$ & $2.57 \mathrm{E}-01$ & $2.50 \mathrm{E}-01$ & I \\
\hline 19 & SSTISAGE1CJ044839.92-692023.4 & 0.18 & 114 & 9.55 & 2.80 & $5.97 \mathrm{E}+03$ & $5.78 \mathrm{E}+03$ & $1.85 \mathrm{E}-04$ & $5.99 \mathrm{E}-04$ & $2.66 \mathrm{E}-01$ & $3.56 \mathrm{E}-01$ & I \\
\hline 20 & SSTISAGE1CJ044847.72-691248.1 & 0.01 & 284 & 6.64 & 0.87 & $1.49 \mathrm{E}+03$ & $8.35 \mathrm{E}+02$ & $1.24 \mathrm{E}-05$ & $7.94 \mathrm{E}-05$ & $6.48 \mathrm{E}-02$ & $8.46 \mathrm{E}-02$ & I \\
\hline 21 & SSTISAGE1CJ044854.41-690948.1 & 0.50 & 49 & 18.62 & 2.26 & $4.25 \mathrm{E}+04$ & $1.27 \mathrm{E}+04$ & $1.06 \mathrm{E}-03$ & $2.53 \mathrm{E}-03$ & $2.89 \mathrm{E}-01$ & $4.86 \mathrm{E}-01$ & I \\
\hline 22 & SSTISAGE1CJ044858.14-684742.2 & 1.18 & 2 & 15.68 & 2.12 & $8.51 \mathrm{E}+03$ & $3.87 \mathrm{E}+02$ & $1.20 \mathrm{E}-02$ & $2.57 \mathrm{E}-03$ & $4.84 \mathrm{E}-02$ & $4.84 \mathrm{E}-02$ & I \\
\hline
\end{tabular}

Notes.

${ }^{\mathrm{a}} \chi_{\min }^{2}(\mathrm{cpd})$ is a minimum $\chi^{2}$ per datapoint.

b $n_{\text {fits }}$ corresponds to a number of fits with cpd between $\mathrm{cpd}_{\min }$ and $\mathrm{cpd}_{\min }+1$.

(This table is available in its entirety in machine-readable and Virtual Observatory (VO) forms in the online journal. A portion is shown here for guidance regarding its form and content.)

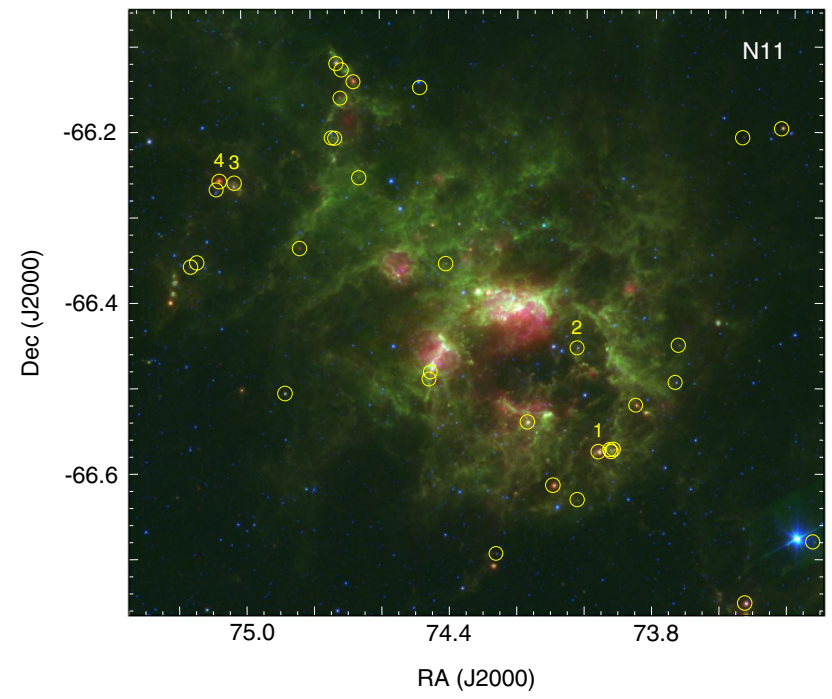

Figure 7. YSOs in the N11 region. The YSO candidates in yellow circles are overlayed on a three-color image ( $24 \mu \mathrm{m}$ : red; $8 \mu \mathrm{m}$ : green; $4.5 \mu \mathrm{m}$ : blue). The yellow circles are large enough to enclose more than one source in some cases, but each is centered on a single YSO (at the IRAC resolution of $\sim 1.5^{\prime \prime}$ ). Sources referred to later in the text are numbered. This also shows the incompleteness of our list which does not include as many sources in regions of very high background or sources extended at IRAC wavelengths.

order of magnitude is useful since this varies by several orders of magnitude for different sources. We do not expect to determine the disk mass well in most of these sources since their SEDs are probably dominated by the envelope emission given their youth.

The $24 \mu \mathrm{m}$ data point helps constrain the longwave shape of the SED and therefore the luminosity of the sources (the integral of the SED), though longer-wave data from both Spitzer and Herschel will improve these estimates. The model fits actually can distinguish stellar temperatures from the near-IR SED, which when combined with luminosity, constrains the stellar mass. Thus, the stellar mass in many of these sources appears fairly well constrained, in the sense that the well-fitted models give similar results. However, we caution that the models assume that the luminosity arises from single sources when in fact many of these are likely multiple. If multiple sources are in the Spitzer beam, the SED will be dominated by the most massive source, if the luminosity is proportional to $\operatorname{mass}^{\alpha}$ and $\alpha$ is greater than 1 . The mass determined from the YSO fits will be slightly higher than the most massive source in the beam, and substantially lower than the total mass of sources in a cluster if they follow a standard IMF. Thus, overall, our mass estimates will be a lower limit by assuming that all of the sources are single.

Figure 15 shows example fits to two YSOs in the N11 region. The source at the left is labeled " 1 " in Figure 7 of the N11 region. The middle and lower panels of Figure 15 show that this source is fitted with an envelope accretion rate of $\sim 4 \times 10^{-4} M_{\odot} \mathrm{yr}^{-1}$, stellar mass $\sim 18 M_{\odot}$, and luminosity $\sim 2.4 \times 10^{4} L_{\odot}$. Note that our accretion rates are model dependent (we assume rotationally flattened free-fall collapse; Ulrich 1976). Nevertheless, the fitted accretion rate corresponds to a fairly massive envelope and suggests that the source is a young protostar. Note that several fits have a disk mass of 0 (left side of left middle panel). All of the models originally had disks, but if the dust sublimation radius is larger than the chosen disk outer radius, the disk mass is set to 0 . This shows that the SEDs of these young sources are not very sensitive to the disk mass.

The source on the right appears more evolved. It lies at the end of an elephant trunk (source "2" in Figure 7. The middleright panel of Figure 15 shows that this sources is fitted as a disk-only source (no infalling envelope). Even for these more evolved sources, the mid-IR is not very sensitive to disk mass, as indicated by the range of values in Figure 15, because the disk is opaque at these wavelengths over a large mass range and thus the emission properties are similar. Longer-wavelength fluxes can better constrain the mass (Robitaille et al. 2007). The mass and luminosity for this source are estimated to be $12 M_{\odot}$ and $1.2 \times 10^{4} L_{\odot}$, respectively.

The three most massive YSOs in Table 5 are source numbers 163, 89, and 625 (SSTISAGE1CJ045854.30-660718.7, SSTISAGE1CJ045358.59-691106.3, and SSTISAGE1CJ052 353.93-713443.7). These all have estimated stellar masses of $\sim 25 M_{\odot}$. All are assigned evolutionary stage I and are therefore young, with envelope infall rates approaching $\sim 10^{-3}-$ $10^{-2} M_{\odot} \mathrm{yr}^{-1}$. The estimated luminosities for these sources are all $\sim 5 \times 10^{4} L_{\odot}$. Note that these are not extraordinarily high mass objects. We are likely missing very high mass objects from our list for two reasons: the most massive sources heat and excite dust out to large radii; for example, Figure 7 shows a few likely YSOs that are extended at IRAC wavelengths and therefore not 


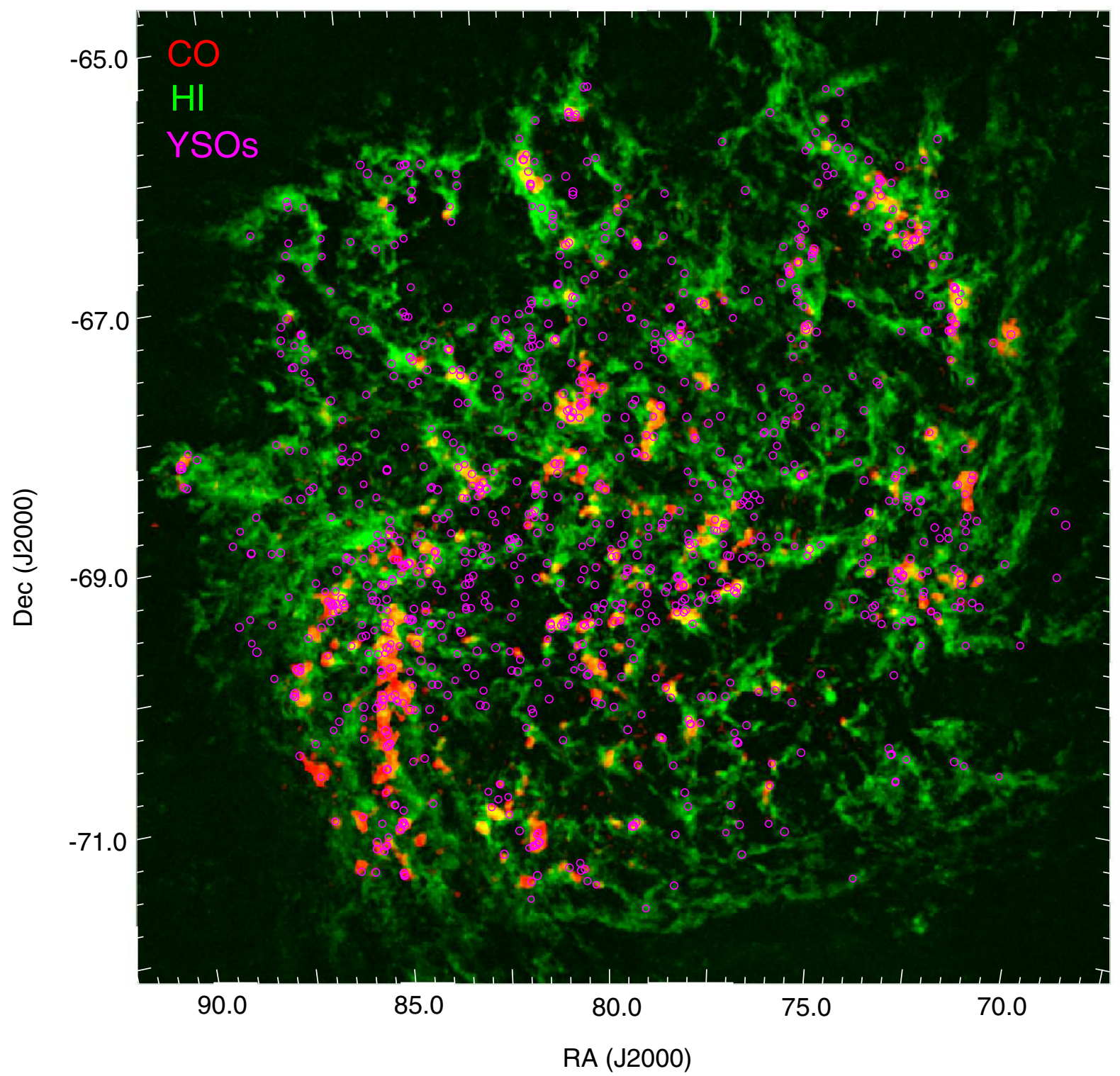

Figure 8. YSOs (magneta circles) overlayed on a two-color image of $\mathrm{H} \mathrm{I}$ gas in green, and $\mathrm{CO}$ gas in red.
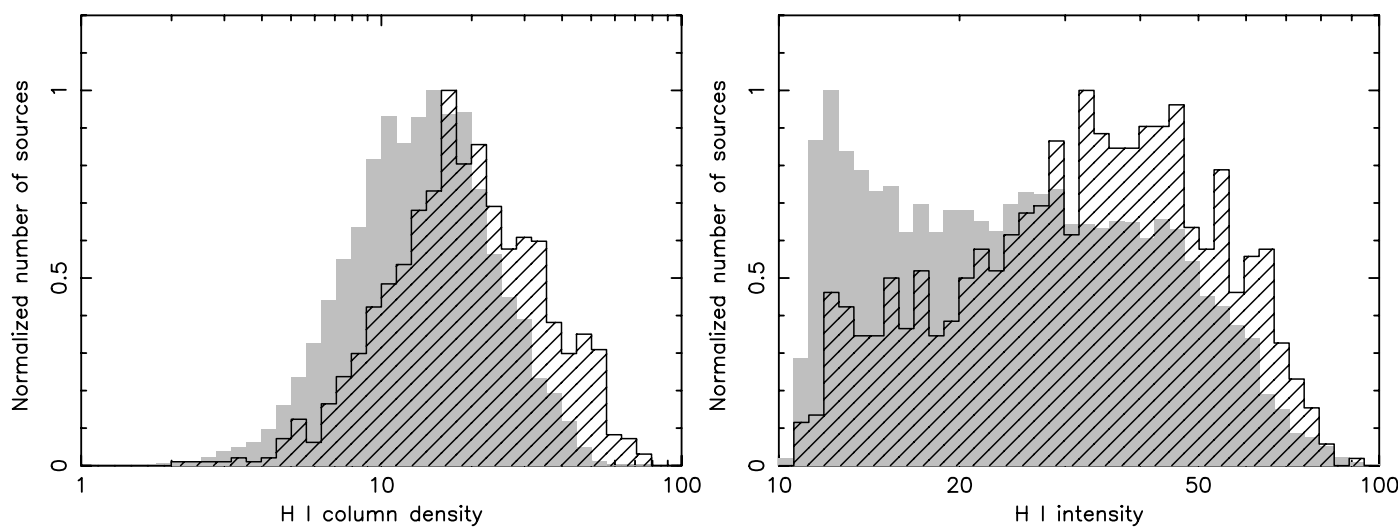

Figure 9. Left: distribution of YSO candidates (hashed) and of the entire SAGE Catalog (gray) as a function of H I column density (in units of $10^{20} \mathrm{~cm}^{-2}$ ). The YSO candidates are associated with higher columns of ISM than those of the SAGE Catalog. Right: distribution of YSO candidates and of the entire SAGE Catalog as a function of $\mathrm{H}$ I peak intensity. 


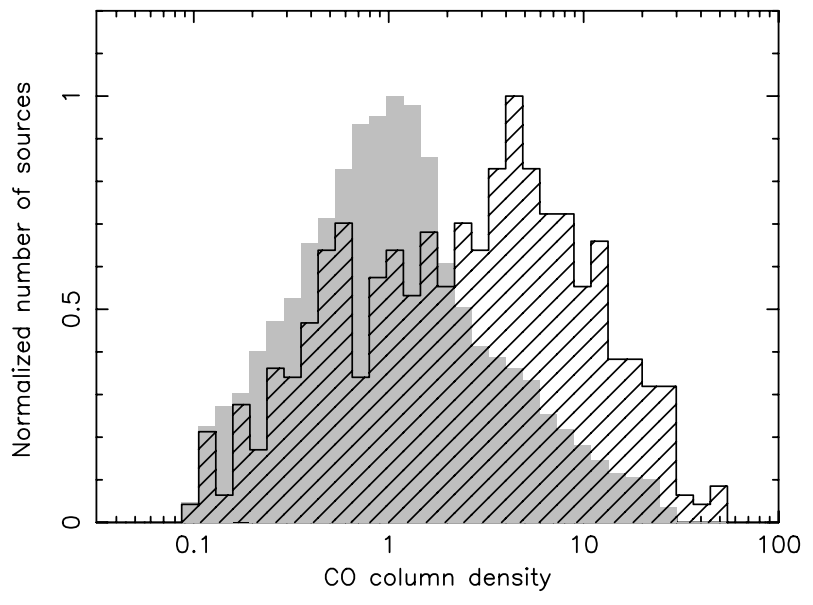

Figure 10. Distribution of YSO candidates (hashed) and of the entire SAGE Catalog (gray) as a function of CO column density (units of $10^{20} \mathrm{~cm}^{-2}$ ).

in our Point Source Catalogs. Second, these massive YSOs may overlap in color-magnitude space with the most luminous AGB stars as shown in Figure 3. This region of color-magnitude space was avoided in our high-probability YSO selection.

The total mass of well-fitted YSOs is estimated to be $2900 M_{\odot}$, and the total luminosity is $2 \times 10^{6} L_{\odot}$. These were calculated by summing the averages of all the well-fitted models. Figure 16 shows histograms of the estimated masses and luminosities of the well-fitted YSOs. Note that some of the number values are less than 1 , because it is a histogram of all the well-fitted distributions (normalized to the number of fits), instead of the average value for each source. The rounded shapes of the histograms show incompleteness at both ends of the mass spectrum. As mentioned previously, we are missing some high-mass sources that are extended at the IRAC wavelengths. Another reason for the fall-off at the high-mass end could be because of the faster evolution expected for the high-mass protostars and thus shorter YSO lifetimes. Our mass function is a YSO mass histogram and not a stellar IMF. And of course we are missing low-luminosity sources due to our selection criteria avoiding the lower-luminosity background galaxies. Our mass function may be complete over the range of $\sim 6-10 M_{\odot}$. We note that the mass estimates assume that a single source produces the measured SED. As noted previously, our mass estimates are a lower limit by assuming that all of the sources are single. All of this points to a likely lower limit in our estimate of YSO masses.

Using the derived parameters to estimate evolutionary stage, Table 5 has 145 Stage I (young embedded) sources, 147 Stage II (optically thick disk) sources, and seven Stage III (optically thin disk) sources. If we had an unbiased list of YSOs, we could estimate evolutionary timescales from the ratios of the numbers at different stages. In the Taurus molecular cloud, for example, there are roughly ten times more Stage II sources than Stage I sources, and therefore the embedded infalling envelope stage is thought to have a lifetime roughly $1 / 10$ th as long as the star+disk stage $\left(10^{5}\right.$ years versus $10^{6}$ years, respectively; Lada 1999). If we assume evolutionary timescales are similar to Taurus, it is clear that our YSO list is biased toward younger sources since the number of Stage I sources is similar to that of the Stage II sources. This is not surprising since we have only selected sources with red [8.0]-[24] colors for this high-probability YSO list.

\subsection{The Current Star-Formation Rate and Efficiency of the $L M C$}

We can calculate the current SFR by estimating the total mass of YSOs and dividing by a formation timescale. To estimate the total mass, we attach a Kroupa (2001) IMF to our mass function over the narrow range that our source list may be complete (Figure 16). Rather than fitting an uncertain slope, we adopt the Kroupa average slopes of -1.3 for $M_{\star}>0.5$, and -0.3 for $M_{\star}<0.5$. Our integration limits went from 0.08 to 50 solar masses. Clearly the choice of both the slope and lower integration limit has a large effect on the total estimated mass. Integrating the Kroupa IMF gives a total mass of YSOs of $11300 M_{\odot}$. We include the masses derived only from the 299 well-fitted high-probability YSOs shown in Table 5. Since most of the YSOs in Table 5 are young and relatively massive, we assume a formation timescale of $\approx 2 \times 10^{5} \mathrm{yr}$. This gives an SFR of $0.06 M_{\odot} \mathrm{yr}^{-1}$. This is likely uncertain by an order of magnitude given our uncertain estimates of both mass and lifetime.

We can compare this estimate to those calculated from integrating global fluxes. The Kennicutt (1998) relations for ultraviolet and far-IR fluxes give $0.08 M_{\odot} \mathrm{yr}^{-1}$ and $0.17 M_{\odot} \mathrm{yr}^{-1}$ respectively, using the rocket ultraviolet image of Smith et al. (1987) and the SAGE MIPS data (at 24, 70, and $160 \mu \mathrm{m}$ ) for the thermal IR flux (see Dale \& Helou 2002). The new calibrations
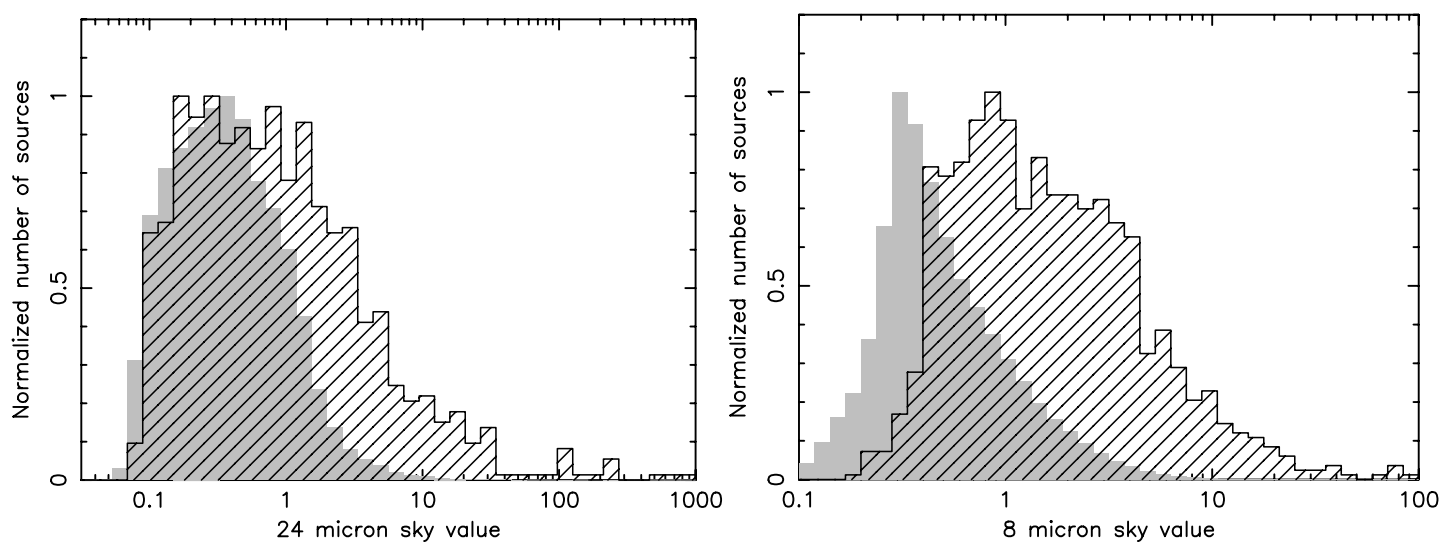

Figure 11. Distribution of YSO candidates (hashed) and of the entire SAGE Catalog (gray) as a function of $24 \mu \mathrm{m}$ intensity (left) and $8 \mu \mathrm{m}$ intensity (right) (units of MJy sr ${ }^{-1}$ ). The main difference between these panels is in the catalog distribution rather than the YSOs. The catalog sources peak in regions of low $8 \mu \mathrm{m}$ emission, in agreement with Figure 6 which shows massive stars inside the $8 \mu \mathrm{m}$ bubbles. 

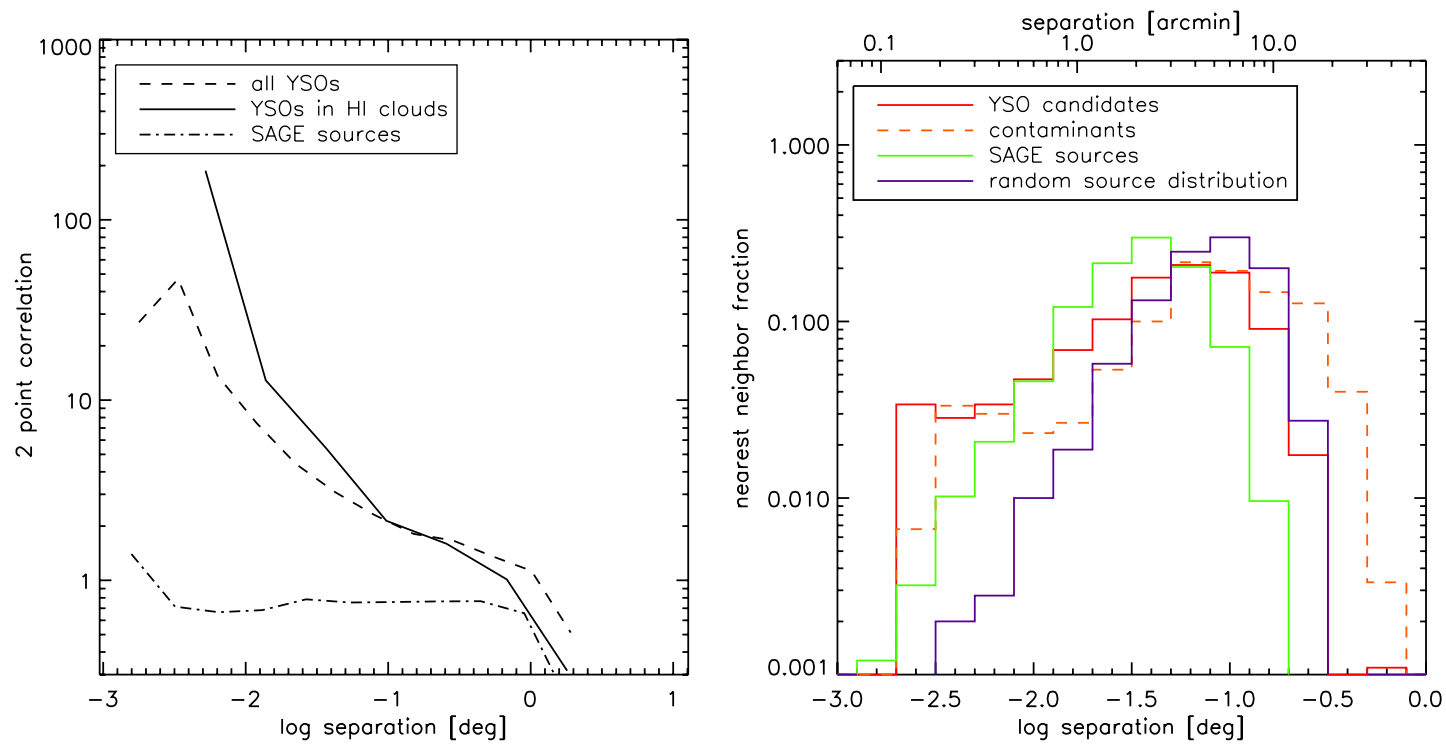

Figure 12. Two measures of clustering of the YSO candidates. The left panel shows the two-point correlation functions of YSOs (dashed and solid lines) and generic SAGE Point Sources (dot-dashed). A break in the correlation function can indicate a preferred scale for star formation, such as a Jeans mass, but the roughly power-law form seen here merely indicates strong hierarchical clustering on all scales. The right panel shows the nearest-neighbor distribution of the relevant populations. An excess at small scales in this distribution can indicate a fragmentation or other preferred clustering scale. LMC YSOs are tightly clustered down to the smallest scales that we can measure $\left(\sim 10^{\prime \prime}\right)$. In the key, "contaminants" refer to the sources identified as non-YSOs.

from Calzetti et al. (2007) give an SFR of $0.05 M_{\odot} \mathrm{yr}^{-1}$ using the SAGE MIPS24 image, and $0.14 M_{\odot} \mathrm{yr}^{-1}$ using the Southern H-Alpha Sky Survey Atlas (SHASSA) images (Gaustad et al. 2001). The combined rates from H-alpha and MIPS24 (Calzetti et al. 2007) give $0.19 M_{\odot} \mathrm{yr}^{-1}$ for stars with age $<10 \mathrm{Myr}$. The ultraviolet and thermal IR combined rates are $0.25 M_{\odot} \mathrm{yr}^{-1}$ for stars $<100 \mathrm{Myr}$ old. These estimates agree with that made by Kennicutt et al. (1995) based on the $\mathrm{H}-\alpha$ luminosity if we use the newer conversion factors (Kennicutt 1998) and make a $0.3 \mathrm{mag}$ correction for extinction.

Gorjian et al. (2004) estimated the SFR of the star-forming region Henize 206 in the LMC, also using the Kennicutt (1998) relations, and calculated a value of $1 \times 10^{-3} M_{\odot} \mathrm{yr}^{-1}$ for an area of $0.021 \mathrm{deg}^{2}$, or $8 \times 10^{-3} M_{\odot} \mathrm{yr}^{-1} \mathrm{kpc}^{-2}$ (assuming a distance to the LMC of $50 \mathrm{kpc}$ ). Indebetouw (2008) estimated the SFR of the southeast (SE) ridge (discussed in Section 3.2) using the Schmitt-Kennicutt law (Kennicutt 1998) and found a value of $0.14 M_{\odot} \mathrm{yr}^{-1} \mathrm{kpc}^{-2}$. A rough estimate of the total star-forming area of the LMC can be made from the extent of the detectable $\mathrm{HI}$ and $70 \mu \mathrm{m}$ emission. From this we estimate the area to be $34 \mathrm{kpc}^{2}$ or $44 \mathrm{deg}^{2}$. Scaling the global value calculated above, we obtain $\approx 5 \times 10^{-3} M_{\odot} \mathrm{yr}^{-1} \mathrm{kpc}^{-2}$. Based on these calculations, the SE ridge does appear to be more active than average, in agreement with models that suggest triggered star formation in this region (de Boer et al. 1998). Simon et al. (2007) calculated the SFR for the N66 region in the SMC, also using YSO SED fitting, and found a rate of $0.066 M_{\odot} \mathrm{yr}^{-1} \mathrm{kpc}^{-2}$ consistent with its being the most active star-formation region in the SMC.

As noted in Section 1, optical CMD modeling has determined historical relative rates of star formation in the LMC (e.g., Gallagher et al. 1996; Olsen 1999; Smecker-Hane et al. 2002). The LMC is thought to be in an active period of star and cluster formation currently (Da Costa 1991; Hodge 1988), with an increase in the SFR from 3-4 Gyr ago by a factor of about 3 (Geha et al. 1998; Holtzman et al. 1997). This is consistent with the new results (Kallivayalil et al. 2006b, 2006a; Besla et al. 2007) suggesting that the LMC is in its first passage with the Milky Way, having entered the virial radius of the dark matter halo 1-3 Gyr ago. The SFR estimated by the YSOs and integrated fluxes set an absolute scale to the star-formation history.

The star-formation efficiency (SFE) can be estimated by dividing the total mass of YSOs by the mass of molecular gas ( $\sim 4 \times 10^{7} M_{\odot}$; Fukui et al. 1999), giving $\sim 3 \times 10^{-4}$, much lower than the estimate of $1 \%$ (Fukui et al. 1999). Their estimate was based on 45 young populous clusters associated with molecular clouds (Bica et al. 1996), assuming a mass of $10^{4} M_{\odot}$ for each, and dividing by the same molecular gas mass (Fukui et al. 1999). The likely reason for the discrepancy is due to the very different lifetimes of YSOs and young clusters. To accurately calculate the SFE, we need to know the molecular cloud lifetimes and the total number of stars produced in that time. The YSO-based calculation is a lower estimate since the time over which the YSOs have strong IR excesses, and are thus selected by the methods in this paper, is likely much shorter than the molecular cloud lifetimes. The cluster estimate is probably more accurate, since the clusters are still associated with the clouds and thus place lower limits on the age of the clouds.

\section{SUMMARY}

Our new YSO candidate list increases the number of suspected YSOs in the LMC from a handful to over a thousand. The list was selected to contain as many YSOs as possible in regions of color-magnitude space relatively free of other populations, but the resulting list likely includes some evolved stars and galaxies. We estimate a conservative upper limit of 55\% contamination by these populations. Follow-up spectroscopic observations will guide us further in separating these populations. Our YSO list will expand as we include extended IRAC sources and long-wave MIPS sources, use future SAGE Catalogs 

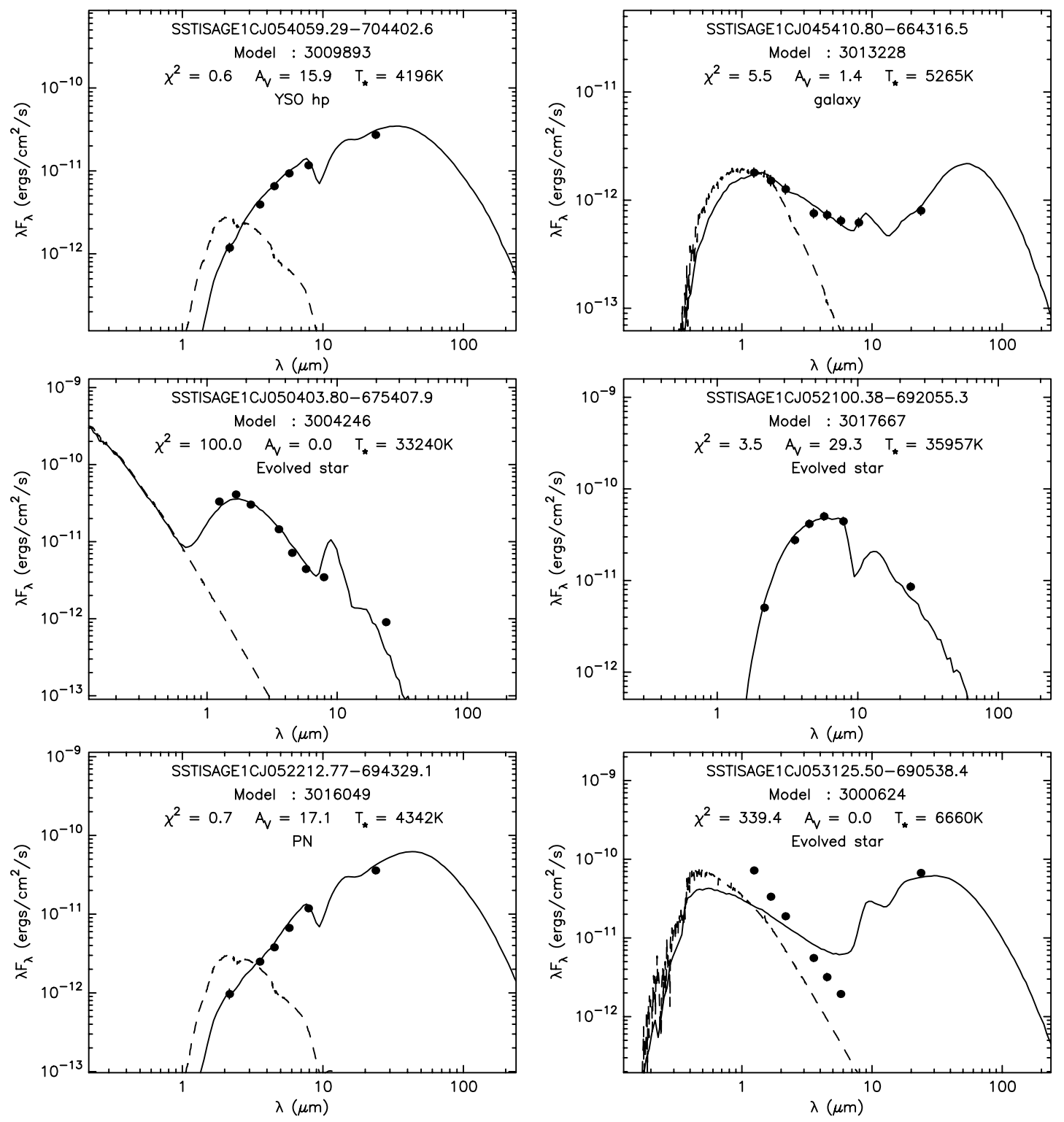

Figure 13. SEDs of sources of different categories. top left: YSO; top right: QSO; middle left: carbon star; middle right: AGB; bottom left: PN; bottom right: WR star. The dashed line in each plot is the central stellar atmosphere extincted by the fitted foreground extinction (shown in the plot).

(An extended figure set is available in the online journal)

that go deeper, employ statistical methods for source classification (Harvey et al. 2007; Marengo et al. 2006), and improve our high-mass YSO radiation transfer models. However, the current list should be of immediate use for follow-up studies. In addition, users can select the 458 "YSO_hp" sources in Table 2 which have a higher probability of being YSOs.

We compared our candidate YSO list to gas tracers ( $\mathrm{HI}$ and $\mathrm{CO}$ ) and found it substantially more correlated than the SAGE Catalog. As expected, the correlation is strongest with molecular gas as traced by $\mathrm{CO}$. The YSOs are correlated with $8 \mu \mathrm{m}$ and $24 \mu \mathrm{m}$ emission in a similar fashion, but the SAGE Catalog sources are correlated significantly more with $24 \mu \mathrm{m}$ emission than $8 \mu \mathrm{m}$ (Figure 11). This agrees with the picture also indicated by Figure 6, in which the massive MS stars are found in the $\mathrm{H}$ II regions inside the shells in which the YSOs are more predominantly found. The $24 \mu \mathrm{m}$ emission is also found in the $\mathrm{H}$ II regions but the $8 \mu \mathrm{m}$ emission is mostly in the shells. The MS stars with ages $<10$ Myr likely produced the shells in which the YSOs are currently forming, in agreement with previous work suggesting self-propagating star formation (Feitzinger et al. 1981; Oey \& Massey 1995; Efremov \& Elmegreen 1998a).

The YSOs are more clustered than generic catalog sources, as shown in Figure 12. The roughly power-law shape of the two-point correlation function indicates similar clustering on all scales (hierarchical), though it is possible that the tail of elevated correlation on size scales of $\sim 450 \mathrm{pc}$ is associated with supershells. The nearest-neighbor distribution shows that YSOs are more clustered than catalog sources down to the smallest scales we can measure, $\sim 3$ pc.

We calculated physical parameters of 299 high-probability YSOs whose SEDs were well fitted by a large grid of radiation transfer models. These include estimates of stellar mass, 

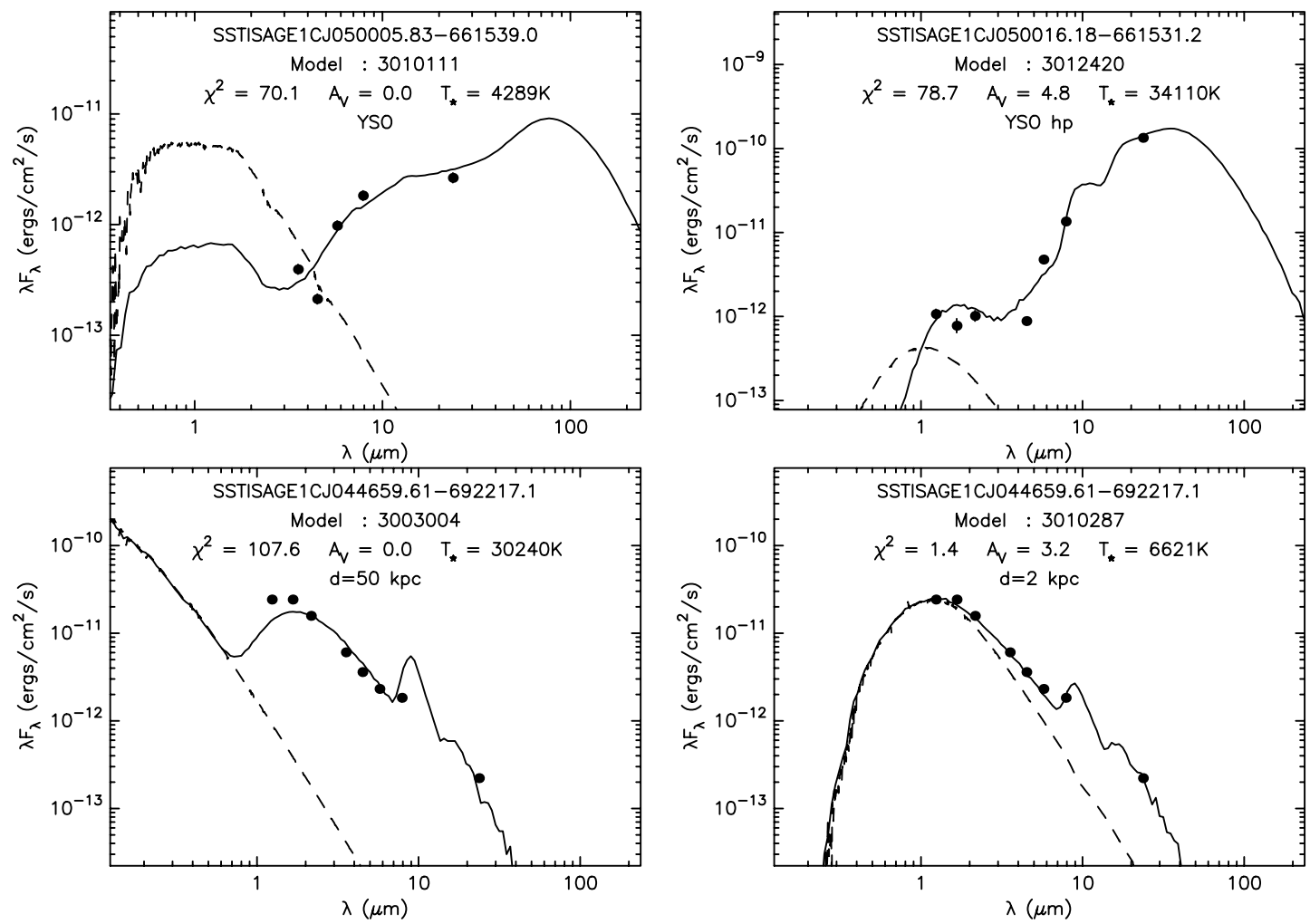

Figure 14. Top: examples of two sources not well fitted by YSO models. Both are likely to be YSOs, but the one at the left probably has PAH emission not accounted for in our models. The one at the right may include more than one source contributing to the excess near-IR emission not normally associated with an embedded protostar. Bottom: at the left is a source poorly fitted by our YSO models fitted with a distance of $50 \mathrm{kpc}$. At the right is the same source fitted well with a distance of only $1.5 \mathrm{kpc}$. The stellar photospheres are shown as dashed lines, extincted by the fitted foreground value. The stellar temperature for the fit at right is about $6500 \mathrm{~K}$, and the IR excess is modest. This is likely an evolved star, as its SED is similar to one shown in Figure 13 (middle-left panel). The evolved-star models can be fitted with LMC distances (A. Ginsburg et al. 2008, in preparation).

envelope infall rate, disk mass, and luminosity (Table 5). The estimated summed mass and luminosity of the YSOs is $\sim 2900 M_{\odot}$ and $\sim 2 \times 10^{6} L_{\odot}$, respectively. Attaching a standard (Kroupa 2001) IMF to the mass histogram over the mass range thought to be complete $\left(6-10 M_{\odot}\right)$ and integrating gives a total mass of YSOs in the LMC of $11300 M_{\odot}$. About half of the sources are classified as Stage I, or young sources, still surrounded by infalling envelopes. This is not surprising because our colormagnitude selection criteria was biased toward redder, and therefore younger sources.

The SFR of the LMC was calculated by dividing the total estimated mass of the subset of YSOs (the well-fitted highprobability YSOs) by their estimated ages, giving $\sim 0.06 M_{\odot}$ $\mathrm{yr}^{-1}$ but the uncertainty of this estimate is likely an order of magnitude, due to our incomplete source list, uncertain IMF, and uncertain age estimate for the YSOs. In the future, as our YSO Catalog becomes more complete, the SFR estimates will become more accurate. The estimate based on YSO counting is lower than most of the estimates calculated from the integrated ultraviolet, $\mathrm{H}-\alpha$, and IR fluxes from the LMC, which give $\sim 0.05-0.25 M_{\odot} \mathrm{yr}^{-1}$. Compared to the Milky Way Galaxy, which has a SFR of $\sim 5 M_{\odot} \mathrm{yr}^{-1}$ (Smith et al. 1978; Diehl et al. 2006) and a total mass $\sim 50$ times greater than the LMC (van der Marel et al. 2002; Besla et al. 2007), the LMC SFR is comparable or slightly larger than the Galactic rate. Compared to the SMC, which has an estimated SFR of $0.05 M_{\odot} \mathrm{yr}^{-1}$ (Wilke et al. 2004) and a mass of 5-10 times smaller than the LMC (Besla et al. 2007), the SMC appears to be more active than the LMC at the present time.
To accurately estimate the SFE, we would count all the stars formed during the lifetime of the molecular cloud. Our SFE estimate of $3 \times 10^{-4}$, based on the ratio of YSO masses to molecular cloud mass, is a lower limit since the age of the YSOs is likely much younger than the age of the molecular clouds; i.e., those YSOs that have evolved into MS stars during the lifetime of the cloud are not being counted. Fukui et al. (1999)'s estimate of 0.01 could be an upper limit if the ages of the associated young clusters is larger than the molecular cloud lifetime, though it is certainly more accurate than the YSO estimate. A better estimate of cloud lifetimes in conjunction with star-formation history models would improve the SFE estimate. Future work based on the SAGE data (T. Onishi et al. 2008, in preparation) will better determine cloud lifetimes by determining the number of clouds with and without star-formation activity.

We thank the anonymous referee whose comments helped improve this paper. We thank Eva Villaver for providing helpful information about PNe. This work was supported by NASA grants to the Spitzer SAGE Legacy project (1275598, 1275588, $1282646,1275421,397380,1275471$, and 1282688), the Spitzer Theoretical Research Program (1290701; B. W., T. R.), the Spitzer Fellowship Program (R. I.), the NASA Theory Program (NNG05GH35G; B.W.), NASA grant NAG5-12595 (M. M., U. V.). This research is based on observations made with the Spitzer Space Telescope, which is operated by the Jet Propulsion Laboratory, California Institute of Technology under a contract with NASA; has made use of data products from the 2MASS, 

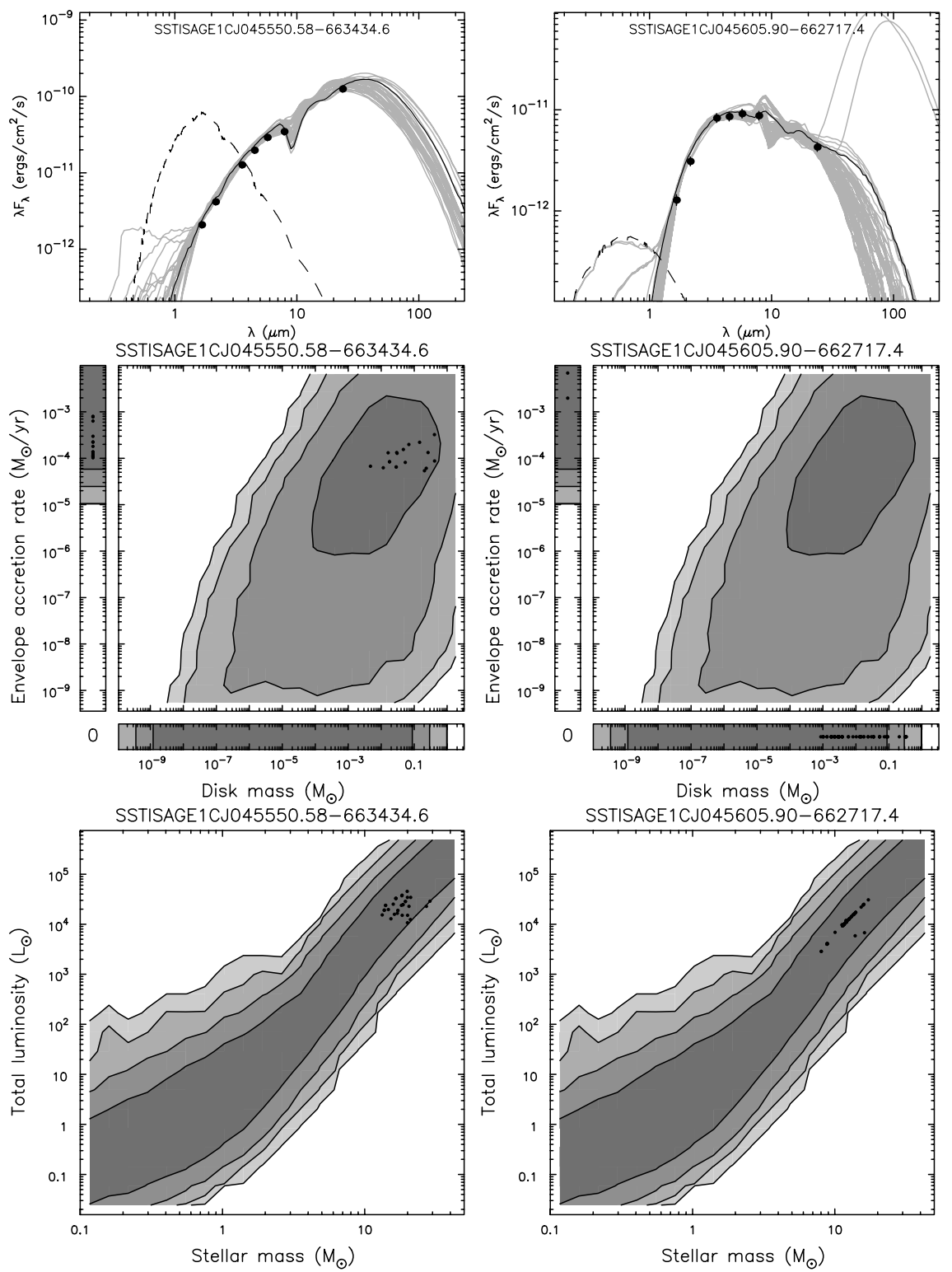

Figure 15. Example YSO fitter results on two sources. These are sources 1 and 2 in Figure 7. Top: the gray lines show all the models with $\chi^{2}$ per data point between the minimum value, $\mathrm{cpd}_{\min }$, and $\mathrm{cpd}_{\min }+1$. The dashed line shows the input stellar atmosphere file extincted by the fitted foreground value. The middle and bottom panels show different model parameters for the same set of model fits as the top panel. The contoured gray scale shows how the parameters of the entire model grid are distributed. The source at left is fitted with a substantial envelope and the source at right is fitted with disk-only models and is therefore likely more evolved.
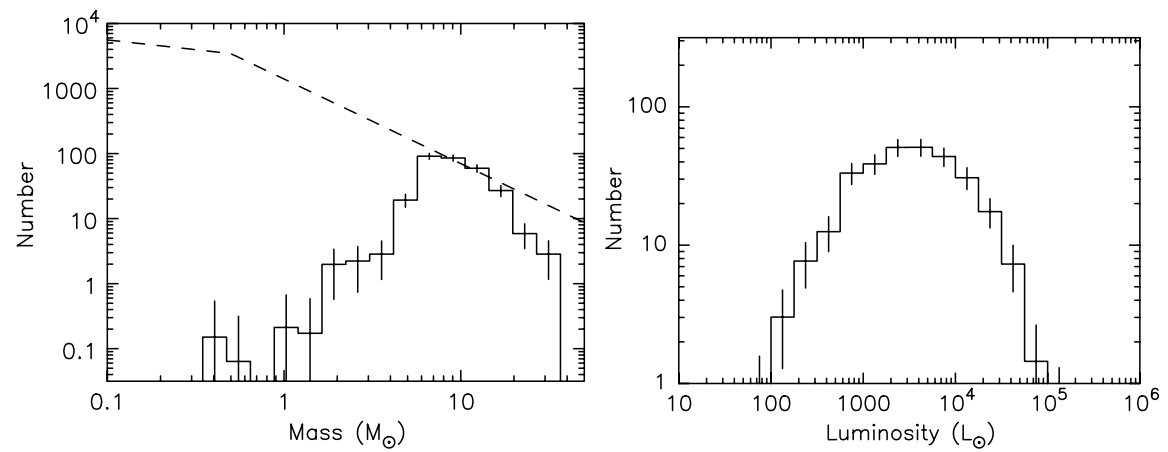

Figure 16. Histograms of stellar mass and luminosity for the well-fitted high-probability YSO candidates (Table 5). The dashed line in the left panel is the (Kroupa 2001) IMF (see Section 3.5). 
which is a joint project of the University of Massachusetts and the IR Processing and Analysis Center/California Institute of Technology, funded by NASA and the National Science Foundation; and has used the SIMBAD database, operated at CDS, Strasbourg, France.

\section{REFERENCES}

Besla, G., Kallivayalil, N., Hernquist, L., Robertson, B., Cox, T. J., van der Marel, R. P., \& Alcock, C. 2007, ApJ, 668, 949

Bica, E., Claria, J. J., Dottori, H., Santos, J. F. C. Jr., \& Piatti, A. E. 1996, ApJS, 102,57

Blum, R. D., et al. 2006, AJ, 132, 2034

Bohannan, B., \& Epps, H. W. 1974, A\&AS, 18, 47

Bolatto, A. D., et al. 2007, ApJ, 655, 212

Brandner, W., Grebel, E. K., Barbá, R. H., Walborn, N. R., \& Moneti, A. 2001, AJ, 122, 858

Breysacher, J., Azzopardi, M., \& Testor, G. 1999, A\&AS, 137, 117

Brott, I., \& Hauschildt, P. H. 2005, in ESA SP-576, The Three-Dimensional Universe with Gaia, ed. C. Turon, K. S. O Flaherty, \& M. A. C. Perryman, (Noordwijk: ESA), 565

Buchanan, C. L., Kastner, J. H., Forrest, W. J., Hrivnak, B. J., Sahai, R., Egan, M., Frank, A., \& Barnbaum, C. 2006, AJ, 132, 1890

Calzetti, D., et al. 2005, ApJ, 633, 871

Calzetti, D., et al. 2007, ApJ, 666, 870

Chu, Y.-H., et al. 2005, ApJ, 634, L189

Cioni, M.-R. L., Girardi, L., Marigo, P., \& Habing, H. J. 2006, A\&A, 448, 77

Cohen, M., et al. 2007, MNRAS, 374, 979

Da Costa, G. S. 1991, in IAU Symp. 148, The Magellanic Clouds, ed. R. Haynes, \& D. Milne, (Dordrecht: Kluwer), 183

Dale, D. A., \& Helou, G. 2002, ApJ, 576, 159

de Boer, K. S., Braun, J. M., Vallenari, A., \& Mebold, U. 1998, A\&A, 329, L49

de Wit, W. J., Beaulieu, J. P., \& Lamers, H. J. G. L. M. 2002, A\&A, 395, 829

de Wit, W. J., Beaulieu, J. P., Lamers, H. J. G. L. M., Coutures, C., \& Meeus, G. 2005, A\&A, 432, 619

Diehl, R., et al. 2006, Nature, 439, 45

Diolaiti, E., Bendinelli, O., Bonaccini, D., Close, L., Currie, D., \& Parmeggiani, G. 2000, A\&AS, 147, 335

Draine, B. T. 2003, ARA\&A, 41, 241

Efremov, Y. N., \& Elmegreen, B. G. 1998a, MNRAS, 299, 588

Efremov, Y. N., \& Elmegreen, B. G. 1998b, MNRAS, 299, 643

Egan, M. P., Van Dyk, S. D., \& Price, S. D. 2001, AJ, 122, 1844

Ellingsen, S. P., Whiteoak, J. B., Norris, R. P., Caswell, J. L., \& Vaile, R. A. 1994, MNRAS, 269, 1019

Epchtein, N., Braz, M. A., \& Sevre, F. 1984, A\&A, 140, 67

Fazio, G. G., et al. 2004, ApJS, 154, 10

Feitzinger, V., Glassgold, A. E., Gerola, H., \& Seiden, P. E. 1981, A\&A, 98, 371

Fukui, Y., et al. 1999, PASJ, 51, 745

Gallagher, J. S., et al. 1996, ApJ, 466, 732

Gatley, I., Becklin, E. E., Hyland, A. R., \& Jones, T. J. 1981, MNRAS, 197, 17P

Gaustad, J. E., McCullough, P. R., Rosing, W., \& Van Buren, D. 2001, PASP, 113,1326

Geha, M. C., et al. 1998, AJ, 115, 1045

Gibb, E. L., et al. 2000, ApJ, 536, 347

Giveon, U., Richter, M. J., Becker, R. H., \& White, R. L. 2007, AJ, 133, 639

Gordon, K. D., et al. 2005, PASP, 117, 503

Gorjian, V., et al. 2004, ApJS, 154, 275

Gouliermis, D., Brandner, W., \& Henning, T. 2006, ApJ, 636, L133

Hartmann, L., Ballesteros-Paredes, J., \& Bergin, E. A. 2001, ApJ, 562, 852

Harvey, P., Merín, B., Huard, T. L., Rebull, L. M., Chapman, N., Evans, N. J.

II, \& Myers, P. C. 2007, ApJ, 663, 1149

Hodge, P. 1988, PASP, 100, 1051

Hollenbach, D., Johnstone, D., Lizano, S., \& Shu, F. 1994, ApJ, 428, 654

Holtzman, J. A., et al. 1997, AJ, 113, 656

Hora, J. L., et al. 2008, AJ, 135, 726

Hyland, A. R., Straw, S., Jones, T. J., \& Gatley, I. 1992, MNRAS, 257, 391

Indebetouw, R. 2008, ApJ, submitted

Indebetouw, R., Johnson, K. E., \& Conti, P. 2004, AJ, 128, 2206

Indebetouw, R., Robitaille, T. P., Whitney, B. A., Churchwell, E., Babler, B., Meade, M., Watson, C., \& Wolfire, M. 2007, ApJ, 666, 321

Indebetouw, R., et al. 2005, ApJ, 619, 931

Israel, F. P., et al. 2003, A\&A, 406, 817

Jones, T. J., Hyland, A. R., Straw, S., Harvey, P. M., Wilking, B. A., Joy, M., Gatley, I., \& Thomas, J. A. 1986, MNRAS, 219, 603

Jones, T. J., Woodward, C. E., Boyer, M. L., Gehrz, R. D., \& Polomski, E. 2005, ApJ, 620, 731
Kallivayalil, N., van der Marel, R. P., \& Alcock, C. 2006a, ApJ, 652, 1213

Kallivayalil, N., van der Marel, R. P., Alcock, C., Axelrod, T., Cook, K. H., Drake, A. J., \& Geha, M. 2006b, ApJ, 638, 772

Keane, J. V., Tielens, A. G. G. M., Boogert, A. C. A., Schutte, W. A., \& Whittet, D. C. B. 2001, A\&A, 376, 254

Kennicutt, R. C. Jr. 1998, ARA\&A, 36, 189

Kennicutt, R. C. Jr., Bresolin, F., Bomans, D. J., Bothun, G. D., \& Thompson, I. B. 1995, AJ, 109, 594

Kontizas, E., Dapergolas, A., Morgan, D. H., \& Kontizas, M. 2001, A\&A, 369,932

Kroupa, P. 2001, MNRAS, 322, 231

Kurucz, R. 1993, ATLAS9 Stellar Atmosphere Programs and $2 \mathrm{~km} \mathrm{~s}^{-1}$ Grid Kurucz CD-ROM No. 13 (Cambridge, MA: Smithsonian Astrophysical Obs.) 13

Kwok, S. 1993, ARA\&A, 31, 63

Lada, C. J. 1999, in NATO ASIC Proc. 540, The Origin of Stars and Planetary Systems, ed. C. J. Lada, \& N. D. Kylafis, (Dordrecht: Kluwer), 143

Lamers, H. J. G. L. M., Beaulieu, J. P., \& de Wit, W. J. 1999, A\&A, 341, 827

Leisy, P., Dennefeld, M., Alard, C., \& Guibert, J. 1997, A\&AS, 121, 407

Lonsdale, C., et al. 2004, ApJS, 154, 54

Luks, T., \& Rohlfs, K. 1992, A\&A, 263, 41

Madau, P., Ferguson, H. C., Dickinson, M. E., Giavalisco, M., Steidel, C. C., \& Fruchter, A. 1996, MNRAS, 283, 1388

Marengo, M., Allen, L. E., Ashby, M. L., Patten, B. M., Sanchez, M. C., \& IRAC Shallow Survey Collaboration. 2006, in AAS Meeting Abstracts Vol. 209, \#161.07 available at http://www.lpi.usra.edu/meetings/ppv2005/pdf/8566.pdf Meixner, M., et al. 2006, AJ, 132, 2268

Mizuno, N., et al. 2001, PASJ, 53, 971

Oey, M. S., \& Massey, P. 1995, ApJ, 452, 210

Oliveira, J. M., van Loon, J. T., Stanimirović, S., \& Zijlstra, A. A. 2006, MNRAS, 372, 1509

Olsen, K. A. G. 1999, AJ, 117, 2244

Panagia, N. 2005, in IAU Colloq. 192, Cosmic Explosions, On the 10th Anniversary of SN1993J, ed. J.-M. Marcaide, \& K. W. Weiler, (Berlin: Springer), 585

Panagia, N., Romaniello, M., Scuderi, S., \& Kirshner, R. P. 2000, ApJ, 539, 197

Pei, Y. C., Fall, S. M., \& Hauser, M. G. 1999, ApJ, 522, 604

Porras, A., et al. 2007, ApJ, 656, 493

Reid, W. A., \& Parker, Q. A. 2006, MNRAS, 373, 521

Rieke, G. H., et al. 2004, ApJS, 154, 25

Robberto, M., Beckwith, S. V. W., \& Panagia, N. 2002, ApJ, 578, 897

Robitaille, T. P., Whitney, B. A., Indebetouw, R., \& Wood, K. 2007, ApJS, 169,328

Robitaille, T. P., Whitney, B. A., Indebetouw, R., Wood, K., \& Denzmore, P. 2006, ApJS, 167, 256

Romaniello, M., Scuderi, S., Panagia, N., Salerno, R. M., \& Blanco, C. 2006, A\&A, 446, 955

Rubio, M., Roth, M., \& Garcia, J. 1992, A\&A, 261, L29

Scalise, E. Jr., \& Braz, M. A. 1982, AJ, 87, 528

Schoenberner, D. 1981, A\&A, 103, 119

Schoenberner, D. 1983, ApJ, 272, 708

Shepherd, D. S., et al. 2007, ApJ, 669, 464

Simon, J. D., et al. 2007, ApJ, 669, 327

Skrutskie, M. F., et al. 2006, AJ, 131, 1163

Smecker-Hane, T. A., Cole, A. A., Gallagher, J. S. III, \& Stetson, P. B. 2002, ApJ, 566,239

Smith, A. M., Cornett, R. H., \& Hill, R. S. 1987, ApJ, 320, 609

Smith, L. F., Mezger, P. G., \& Biermann, P. 1978, A\&A, 66, 65

Srinivasan, S., et al. 2008, AJ, submitted

Staveley-Smith, L., Kim, S., Calabretta, M. R., Haynes, R. F., \& Kesteven, M. J. 2003, MNRAS, 339, 87

Stetson, P. B. 1987, PASP, 99, 191

Teixeira, P. S., et al. 2006, ApJ, 636, L45

Testor, G., Lemaire, J. L., Field, D., \& Diana, S. 2006, A\&A, 453, 517

Ulrich, R. K. 1976, ApJ, 210, 377

van der Marel, R. P., Alves, D. R., Hardy, E., \& Suntzeff, N. B. 2002, AJ, 124,2639

van der Marel, R. P., \& Cioni, M.-R. L. 2001, AJ, 122, 1807

van der Veen, W. E. C. J., Habing, H. J., \& Geballe, T. R. 1989, A\&A, 226, 108

van Loon, J. T., Groenewegen, M. A. T., de Koter, A., Trams, N. R., Waters, L. B. F. M., Zijlstra, A. A., Whitelock, P. A., \& Loup, C. 1999, A\&A, 351, 559 van Loon, J. T., et al. 2005, MNRAS, 364, L71

Volk, K. 1992, ApJS, 80, 347

Walborn, N. R., Drissen, L., Parker, J. W., Saha, A., MacKenty, J. W., \& White, R. L. 1999, AJ, 118, 1684

Watson, C., et al. 2008, ApJ, in press 
Weingartner, J. C., \& Draine, B. T. 2001, ApJ, 548, 296

Werner, M. W., et al. 2004, ApJS, 154, 1

Westerlund, B. E. 1997, The Magellanic Clouds, (New York: Cambridge Univ. Press)

Whiteoak, J. B., Wellington, K. J., Jauncey, D. L., Gardner, F. F., Forster, J. R., Caswell, J. L., \& Batchelor, R. A. 1983, MNRAS, 205, 275

Whitney, B. A., Wood, K., Bjorkman, J. E., \& Cohen, M. 2003a, ApJ, 598, 1079
Whitney, B. A., Wood, K., Bjorkman, J.E., \& Wolff, M. J. 2003b, ApJ, 591, 1049 Wichmann, R., Schmitt, J. H. M. M., \& Krautter, J. 2001, A\&A, 380, L9

Wilke, K., Klaas, U., Lemke, D., Mattila, K., Stickel, M., \& Haas, M. 2004, A\&A, 414, 69

Yamaguchi, R., et al. 2001, PASJ, 53, 985

Zaritsky, D., \& Harris, J. 2004, ApJ, 604, 167

Zaritsky, D., Harris, J., Thompson, I. B., \& Grebel, E. K. 2004, AJ, 128, 1606 